\title{
ON AZUMA-TYPE INEQUALITIES FOR BANACH SPACE-VALUED MARTINGALES
}

\author{
MANUSCRIPT \\ Sijie Luo \\ Yau Mathematical Sciences Center \\ Tsinghua University \\ luosijie@mail.tsinghua.edu.cn
}

\begin{abstract}
In this paper, we will study concentration inequalities for Banach space-valued martingales. Firstly, we prove that a Banach space $X$ is linearly isomorphic to a $p$-uniformly smooth space $(1<p \leq 2)$ if and only if an Azuma-type inequality holds for $X$-valued martingales. This can be viewed as a generalization of Pinelis' work on Azuma inequality for martingales with values in 2-uniformly smooth space. Secondly, Azuma-type inequality for self-normalized sums will be presented. Finally, some further inequalities for Banach space-valued martingales, such as moment inequalities for double indexed dyadic martingales and the De la Peña-type inequalities for conditionally symmetric martingales, will also be discussed.
\end{abstract}

Keywords Azuma inequality $\cdot$ conditionally symmetric martingales $\cdot$ self-normalized sums · uniformly smooth Banach spaces

\section{Introduction}

Concentration inequalities for sums of independent random variables and their extension to martingales have been extensively studied by many authors, such as Bernstein (1927), Kolomogrov (1929), Bennett (1962), Hoeffding (1963), Azuma (1967) etc. To state one, we begin with the following Hoeffding-Azuma inequality, which was proved by Hoeffding [12] for sums of independent bounded random variables and was extended to martingales by Azuma [2].

Theorem 1.1 (Hoeffding-Azuma). Let $\left(f_{j}\right)_{j=0}^{n}$ be a real-valued martingale such that $\left|f_{j}-f_{j-1}\right| \leq a_{j}$ a.s. for all $j=1,2, \cdots n$. Then, for all $r \geq 0$,

$$
\mathbb{P}\left\{\left|f_{n}-f_{0}\right| \geq r\right\} \leq 2 \exp \left\{-\frac{r^{2}}{2\left(a_{1}^{2}+\cdots+a_{n}^{2}\right)}\right\} .
$$

The Hoeffding-Azuma inequality now becomes a basic methodology for proving concentration inequalities for martingales with bounded differences. For some interesting extensions of the method of bounded differences we refer to the survey paper by Godbole and Hitczenko [10]. On the other hand, the Hoeffding-Azuma inequality has been used as a powerful tool in various areas such as probability theory, graph theory, information theory, computer science and related fields (see e.g. [7, 11, 19, 27]).

Recently, motivated by the study of random matrices, concentration inequalities for sums of independent matrix-valued random variables and their extension to matrix-valued martingales have attracted more and more attention. Numerous concentration inequalities have been extended to random variables with values in the space of matrices equipped with operator norm. Several work in this direction have been done by Oliveira [21] Ahlswede and Winter [1], Tropp [28] etc. With the help of the non-commutative generalizations of classical concentration inequalities, fundamental theorems of random matrices have been established successfully. In the matrix-valued setting, deep theory from operator theory, such as the Lieb's concavity theorem [18] and the Golden-Thompson inequality, will be used to derived concentration inequalities. For further information on matrix-valued concentration inequalities we refer to [29] and the extensive references therein. 
Motivated by the study of probability in Banach spaces, it is natural to consider concentration inequalities for Banach space-valued random variables. However, while dealing inequalities in this setting, the main obstacle for proving such inequalities is that the moment generating function and the cumulant generating function methods are not available. To overcome the difficulty, some new techniques must be needed and it will be no surprised that the techniques are not from probabilistic but come from analysis. A great deal of effort has been expended on the study of moment inequalities and integrability of sums of independent Banach space-valued random variables and remarkable results have been established by Ledoux, Talagrand, Maurey, Pisier and many others (see e.g.[17, 26]). However, to the best of our knowledge, the study of concentration inequalities for Banach space-valued random variables is not so widely explored.

In his fundamental work, Naor [20, Theorem 1.5] established the following Azuma inequality for martingales with values in $p$-uniformly smooth Banach space $(1<p \leq 2)$ and then he applied the inequality to obtain an improved estimate of the Alon-Roichman theorem for random Cayley graphs of Abelian groups. Before stating the Naor's result precisely, we shall now recall the $p$-uniform smoothness of Banach spaces.

For a Banach space $X$, the following quantity is called the modulus of uniform smoothness of $X$, for $\tau>0$

$$
\rho_{X}(\tau)=\sup \left\{\frac{\|x+\tau y\|+\|x-\tau y\|}{2}-1: x, y \in X,\|x\|=\|y\|=1\right\} .
$$

Then $X$ is said to be uniformly smooth if $\lim _{\tau \rightarrow 0^{+}} \frac{\rho_{X}(\tau)}{\tau}=0$ and if moreover there exists a constant $s>0$ such that for all $\tau>0$ we have $\rho_{X}(\tau) \leq s \tau^{p}$, then $X$ is said to be $p$-uniformly smooth. It is clear that the $p$-uniform smoothness are only available for $p \in(1,2]$ and, by the parallelogram law (i.e. $|x+y|^{2}+|x-y|^{2}=2\left(|x|^{2}+|y|^{2}\right)$ for all $x, y \in \mathcal{H}$ ), Hilbert space $(\mathcal{H},|\cdot|)$ is of 2 -uniformly smooth.

The following theorem, due to Naor, is an Azuma-type inequality for Banach space-valued martingales.

Theorem 1.2. ([20]) There exists a universal constant $c \in(0, \infty)$ with the following property. Fix $s>0$ and assume that a Banach space $(X,\|\cdot\|)$ satisfies $\rho_{X}(\tau) \leq s \tau^{2}$ for all $\tau>0$. Fix also a sequence of positive numbers $\left(a_{j}\right)_{j=1}^{\infty} \subseteq(0, \infty)$. Let $\left(f_{j}\right)_{j=1}^{\infty}$ be an $X$-valued martingale satisfying $\left\|f_{j}-f_{j-1}\right\| \leq a_{j} a . s$. for all $j \in \mathbb{N}$. Then, for every $r \geq 0$ and $n \in \mathbb{N}$, we have

$$
\mathbb{P}\left\{\left\|f_{n}-f_{0}\right\| \geq r\right\} \leq e^{s+2} \cdot \exp \left\{-\frac{c r^{2}}{a_{1}^{2}+\cdots+a_{n}^{2}}\right\} .
$$

As stated in [20, p. 626], Naor further pointed out: “All our results carry over, with obvious modifications, to general uniformly smooth spaces (of course, in this more general setting, the probabilistic bounds that we get will no longer sub-Gaussian)". Indeed, the main ingredient for proving (1.2) is the [20, Lemma 2.2], which sates that if a 2-uniformly smooth space $X$ with the modulus of uniform smoothness satisfies that $\rho_{X}(\tau) \leq s \tau^{2}$ for all $\tau>0$, then, for every $x$, $y \in X$ and every $q \geq 2$ we have

$$
\frac{\|x+y\|^{q}+\|x-y\|^{q}}{2} \leq\left(\|x\|^{2}+8(s+q)\|y\|^{2}\right)^{q / 2} .
$$

The proof of this lemma can be generalized to $p$-uniformly smooth spaces by replacing 2 of (1.3) with $p$ for all $q \geq p$. Combining with this modification and the Naor's proof of Theorem 1.2 one could deduce the corresponding Azumatype inequality for $p$-uniform smooth space-valued martingales $(1<p \leq 2)$ by replacing $r^{2}$ and $a_{1}^{2}+\cdots+a_{n}^{2}$ on the right hand side of (1.2) by $r^{p}$ and $a_{1}^{p}+\cdots+a_{n}^{p}$ respectively.

However, constants in (1.2) are worse than those in (1.1) and it seems that Naor's method could not lead to better constants even for Hilbert space-valued martingales or certain sub-classes of martingales. Hence, a natural question we consider most is about providing a more precise version of the Azuma-type inequality for martingales with values in $p$-uniformly smooth space $X$, such that, when the Banach space $X$ is a Hilbert space, our results recover the classical Azuma inequality. On the other hand, it is also worthwhile to ask that whether the $p$-uniform smoothness is necessary for Azuma-type inequality to hold. All these questions are natural and worth considering, which could be stated as follows.

- Can we provide an Azuma-type inequality for Banach space-valued martingales with some improvements?

- Is the $p$-uniform smoothness of the image space necessary for Azuma-type inequality?

To answer these questions we found that some improvements of Naor's work must be needed. However, as it will be seen below, the Naor's proof is based on some geometric arguments from Banach space theory and estimation of an 
implicity constant in Pisier's inequality [25], both of which are hard to be improved. Precisely, Naor proved that for martingale $\left(f_{j}\right)_{j=0}^{\infty}$ taking values in 2-uniformly smooth space $X$, then there exists a constant $s>0$ only depending on $X$ such that for every $q \geq 2$ and $n \in \mathbb{N}$ the following inequality holds

$$
\left(\mathbb{E}\left\|f_{n}-f_{0}\right\|^{q}\right)^{\frac{1}{q}} \leq 8 \sqrt{s+q} \cdot \sqrt{\sum_{j=1}^{n}\left(\mathbb{E}\left\|d_{j, f}\right\|^{q}\right)^{\frac{2}{q}}},
$$

where $d_{j, f}=f_{j}-f_{j-1}$ for $j \in \mathbb{N}$. Then, the optimal choice of $q \geq 2$ in (1.4) will yield the desired inequality. However, in order to make optimal choice of $q \geq 2$ of (1.4) to derive Azuma inequality, one needs to involve the $\ell_{\infty}$-norm of the martingale differences $d_{j, f}$. And it seems that even for certain sub-classes of martingales (such as conditionally symmetric martingales) the (1.4) could not lead to a better inequality.

To tackle the problem, our approach is based on a method of Pinelis, who have established some fundamental inequalities for 2-uniformly smooth space-valued martingales (see [22, 23]) and then we apply a "martingale dimension reduction" argument (inspired by [8, Lemma 4.2]) to reduce martingales from $p$-uniformly smooth space-valued to $\mathbb{R}^{2}$-valued. More precisely, we prove the following theorem (corresponds to Corollary 3.4

Theorem 1.3. Let $X$ be a $p$-uniformly smooth space $(1<p \leq 2)$ and $f=\left(f_{j}\right)_{j=0}^{\infty}$ be an $X$-valued martingale such that there exists a non-negative predictable sequence $w=\left(w_{j}\right)_{j=1}^{\infty}$ satisfying $\left\|f_{j}-f_{j-1}\right\| \leq w_{j}$ a.s. for all $j \in \mathbb{N}$. Then, there exists a constant $K$ only depending on $X$ such that for all $r \geq 0$,

$$
\mathbb{P}\left\{f^{*} \geq r\right\} \leq 2 \exp \left\{-\frac{r^{p}}{2 K\left\|\sum_{j=1}^{\infty} w_{j}^{p}\right\|_{\infty}}\right\},
$$

where $f^{*}=\sup _{j \in \mathbb{N}}\left\|f_{j}-f_{0}\right\|$.

This theorem is an improvement of Naor's Azuma inequality for martingales with values in $p$-uniformly smooth space $(1<p \leq 2)$. By the method of "good- $\lambda$ inequality" of Burkholder, we can further deduce that the $p$-uniform smoothness of the image space is necessary for Azuma-type inequalities to hold. Moreover, we found that some other types of inequalities, such as Azuma-type inequality for self-normalized sums, De la Peña inequalities etc. are also true for $p$-uniformly smooth space-valued martingales.

Our paper is organized as follows.

In Section 2 (the next section), preliminary results on martingales theory and geometric properties of uniformly smooth spaces will be collected.

In Section 3, we will focus on the Azuma-type inequalities for martingales with values in $p$-uniformly smooth space. For example, we prove that a martingale $f=\left(f_{j}\right)_{j=0}^{\infty}$ with values in $p$-uniformly smooth space, then an Azuma-type inequality holds for $f=\left(f_{j}\right)_{j=0}^{\infty}$. This can be viewed as an improvement of Naor's Azuma inequality (1.2) and a generalization of Pinelis' Azuma-type inequality for martingales with values in 2-uniformly smooth space (see [22]). Furthermore, by applying the "good- $\lambda$ inequality", we deduce that the $p$-uniform smoothness of the image space is necessary for this type of inequality to hold. Hence, we obtain a characterization of $p$-uniformly smooth spaces in terms of Azuma-type inequalities. At the end of this section, Azuma-type inequality for self-normalized sums will also be discussed.

Section 4 will contain some further inequalities for Banach space-valued martingales. In particular, moment inequalities for Banach space-valued double dyadic martingales and the De la Peña-type inequalites for $p$-uniformly smooth space-valued conditionally symmetric martingales will be presented.

Throughout this paper, $X$ (resp. $\mathcal{H}$ ) stands for separable Banach (resp. Hilbert) space and $X^{*}$ is the dual space of $X$. For constants $K_{1}, K_{2}$, the notation $K_{1}=O\left(K_{2}\right)$ we mean that $K_{1} \leq D K_{2}$ with some universal constant $D>0$. We assume that $\inf \emptyset=\infty$ and $\prod_{j \in \emptyset} u_{j}=1$ for convenience. For random variables $f, g$ on some probability space, we will simply use " $f \leq g$ " to stand that " $f \leq g$ almost surely". For $p \geq 1$, the notation $\left(L^{p}(X),\|\cdot\|_{p}\right)$ stands for the space of all $p$-Bochner integrable $X$-valued functions equipped with the $L^{p}$-norm, that is, $\|f\|_{p}=\left(\int\|f(\omega)\|^{p} d \mathbb{P}(\omega)\right)^{1 / p}$ for all $f \in L^{p}(X)$. For a random process $f=\left(f_{j}\right)_{j=0}^{\infty}$ we simply denote $\sigma\left(f_{0}, \cdots, f_{n}\right)$ the $\sigma$-algebra generated by $f_{0}, \cdots, f_{n}$. 


\section{Preliminaries}

This section will be divided into two parts. The first part includes basic concepts and notations from martingale theory. The second part is a digression to Banach space theory.

\subsection{Basic concepts and results from martingale theory}

The following concepts can be found in any books on probability theory (see e.g. [15]). A sequence of sub- $\sigma$ algebras $\left(\mathscr{F}_{j}\right)_{j=0}^{\infty}$ of a probability space $(\Omega, \mathscr{F}, \mathbb{P})$ is said to be a filtration if $\mathscr{F}_{j-1} \subseteq \mathscr{F}_{j}$ for all $j \in \mathbb{N}$. A sequence $\left(f_{j}\right)_{j=0}^{\infty}$ is called adapted to $\left(\mathscr{F}_{j}\right)_{j=0}^{\infty}$ if $f_{j}$ is $\mathscr{F}_{j}$-measurable for each $j \in \mathbb{N} \cup\{0\}:=\mathbb{N}_{0}$. A random variable $\tau:(\Omega, \mathscr{F}, \mathbb{P}) \rightarrow \mathbb{N} \cup\{\infty\}$ is called a stopping time (relatively to the filtration $\left.\left(\mathscr{F}_{j}\right)_{j=0}^{\infty}\right)$ if $\{\tau=n\} \in \mathscr{F}_{n}$ for each $n \in \mathbb{N}$. Throughout this paper we assume that $\mathscr{F}_{0}=\{\{\emptyset\}, \Omega\}$ for convenience.

A sequence of $X$-valued Bochner-integrable random process $\left(f_{j}\right)_{j=0}^{\infty}$ is called a martingale relatively to the filtration $\left(\mathscr{F}_{j}\right)_{j=0}^{\infty}$, if $\left(f_{j}\right)_{j=0}^{\infty}$ is adapted relatively to the filtration $\left(\mathscr{F}_{j}\right)_{j=0}^{\infty}$ such that $\mathbb{E}\left(f_{j} \mid \mathscr{F}_{s}\right)=f_{s}$ for all $s \leq j$ and $j \in \mathbb{N}$. If it does not cause any confusion, we shall simply call $\left(f_{j}\right)_{j=0}^{\infty}$ a martingale and write $\mathbb{E}_{j}=\mathbb{E}\left(\cdot \mid \mathscr{F}_{j}\right)$ for short. If $\mathbb{E}\left(\left\|f_{j}\right\|^{p}\right)<\infty$ for all $j \in \mathbb{N}(p \geq 1)$ then $\left(f_{j}\right)_{j=0}^{\infty}$ is said to be a $L^{p}$-martingale.

The following theorem is well known fact in probability theory called the "Optional Sampling Theorem"(see [15, p. 209]). Recall that a real-valued adapted random process $f=\left(f_{j}\right)_{j=0}^{\infty}$ is called sub-martingale (resp. super-martingale) if $f_{s} \leq \mathbb{E}_{s}\left(f_{j}\right)$ (resp. $f_{s} \geq \mathbb{E}_{s}\left(f_{j}\right)$ ) for all $s \leq j$.

Theorem 2.1 (Optional Sampling Theorem). Let $f=\left(f_{j}\right)_{j=0}^{\infty}$ be super-martingale and $\sigma \leq \tau$ be stopping times.

i) Assume there exists $T \in \mathbb{N}$ such that $\tau \leq T$ a.s.. Then

$$
f_{\sigma} \geq \mathbb{E}\left(f_{\tau} \mid \mathscr{F}_{\sigma}\right)
$$

and if $f$ is a martingale then the equality holds.

ii) If $f$ is nonnegative and $\tau<\infty$ a.s., then we have $\mathbb{E}\left(f_{\tau}\right) \leq \mathbb{E}\left(f_{0}\right)<\infty, \mathbb{E}\left(f_{\sigma}\right) \leq \mathbb{E}\left(f_{0}\right)<\infty$ and $f_{\sigma} \geq$ $\mathbb{E}\left(f_{\tau} \mid \mathscr{F}_{\sigma}\right)$.

iii) Assume that, more generally, $f$ is only adapted and integrable. Then $f$ is a martingale if and only if $\mathbb{E}\left(f_{\tau}\right)=\mathbb{E}\left(f_{0}\right)$ for any bounded stopping time $\tau$.

For an $X$-valued martingale $f=\left(f_{j}\right)_{j=0}^{\infty}$, the maximal function $f^{*}$ (resp. $f_{n}^{*}$ ) is defined by $f^{*}=\sup _{j \in \mathbb{N}}\left\|f_{j}-f_{0}\right\|$ (resp. $f_{n}^{*}=\max _{1 \leq j \leq n}\left\|f_{j}-f_{0}\right\|$ ). Let $\left(d_{j, f}\right)_{j=1}^{\infty}$ be the martingale differences of $f$, that is, $d_{j, f}=f_{j}-f_{j-1}$ for all $j \in \mathbb{N}$, then define $S_{p}(f)=\left(\sum_{j=1}^{\infty}\left\|d_{j, f}\right\|^{p}\right)^{\frac{1}{p}}\left(\operatorname{resp} . S_{p, n}(f)=\left(\sum_{j=1}^{n}\left\|d_{j, f}\right\|^{p}\right)^{\frac{1}{p}}\right)$.

Recall that a martingale $f$ is said to be conditionally symmetric if and only if

$$
\mathbb{E} \varphi\left(d_{1, f}, \cdots, d_{j-1, f}, d_{j, f}\right)=\mathbb{E} \varphi\left(d_{1, f}, \cdots, d_{j-1, f},-d_{j, f}\right),
$$

for all bounded continuous function $\varphi: X^{j} \rightarrow \mathbb{R}$ and all $j \in \mathbb{N}$. For example, all dyadic martingales are conditionally symmetric.

We now conclude this subsection with an important property of conditionally symmetric martingales (see e.g. [5, p. 53]).

Proposition 2.2. Let $X$ be a Banach space and $f$ be an $X$-valued conditionally symmetric martingale. Then $f$ is also a martingale relatively to the filtration $\left(\mathscr{G}_{j}\right)_{j=0}^{\infty}$ defined by

$$
\mathscr{G}_{j}=\sigma\left(d_{0, f}, \cdots, d_{j, f},\left\|d_{j+1, f}\right\|\right), \forall j \in \mathbb{N}_{0}
$$

where $d_{0, f}:=f_{0}$ for convenience.

\subsection{Digress to Banach space theory}

Recall that a Banach space $X$ is said to be $p$-uniformly smooth if there exists $s>0$ such that

$$
\rho_{X}(\tau) \leq s \tau^{p}
$$

for all $\tau>0$ and denote by $s_{p}(X)$ the infimal constant such that 2.1 holds. It is clear that the $p$-uniform smoothness implies the $q$-uniform smoothness for $q \leq p$, hence, in order to avoid confusion, we say that a Banach space is $p$-uniformly smooth where the $p$ refers to the best possible for which $\rho_{X}(\tau) \leq s \tau^{p}$ holds for each $\tau>0$. 
For a proper extend real-valued lower semicontinuous convex function $h: X \rightarrow \mathbb{R} \cup\{+\infty\}$, the subdifferential of $h$ is a set-valued mapping $\partial h: X \rightarrow 2^{X^{*}}$ defined by, for $x \in X$,

$$
\partial h(x)=\left\{x^{*} \in X^{*}:\left\langle x^{*}, y-x\right\rangle \leq f(y)-f(x), \forall y \in X\right\} .
$$

Definition 2.1. For $p>1$, the generalized dual map $J_{p}: X \rightarrow 2^{X^{*}}$ is defined by

$$
J_{p}(x)=\left\{x^{*} \in X^{*}:\left\langle x^{*}, x\right\rangle=\|x\| \cdot\left\|x^{*}\right\|,\left\|x^{*}\right\|=\|x\|^{p-1}\right\}, x \in X .
$$

The following proposition provides a connection between subdifferential of a convex function and the generalized dual map.

Proposition 2.3. ([6, p. 32]) For $p>1$, the generalized dual map $J_{p}$ is the subdifferential of convex function $\frac{1}{p}\|\cdot\|^{p}$.

We would like to mention here that for a $p$-uniformly smooth Banach space $X(1<p \leq 2)$ the generalized dual map $J_{p}$ is indeed a mapping defined from $X$ to $X^{*}$. In fact, if $X$ is a $p$-uniformly smooth space, then its dual space $X^{*}$ is of $q$-uniformly convex with $\frac{1}{p}+\frac{1}{q}=1$ (see [6, p. 46]). Then, for any $x_{1}^{*}$ and $x_{2}^{*} \in J_{p}(x)$, we have

$$
\left\langle x_{1}^{*}, x\right\rangle=\left\langle x_{2}^{*}, x\right\rangle=\|x\|^{p}
$$

with $\left\|x_{1}^{*}\right\|=\left\|x_{2}^{*}\right\|=\|x\|^{p-1}$. Therefore,

$$
\left\langle\frac{x_{1}^{*}+x_{2}^{*}}{2}, x\right\rangle=\|x\|^{p},
$$

which implies that $\left\|\frac{x_{1}^{*}+x_{2}^{*}}{2}\right\| \geq\|x\|^{p-1}$. On the other hand, by the triangle inequality, we have $\left\|\frac{x_{1}^{*}+x_{2}^{*}}{2}\right\| \leq\|x\|^{p-1}$, which yields that $\left\|\frac{x_{1}^{*}+x_{2}^{*}}{2}\right\|=\|x\|^{p-1}$. Hence, it follows from the uniform convexity of $X^{*}$ that $x_{1}^{*}=x_{2}^{*}$.

The following theorem provides a characterization of $p$-uniformly smooth spaces by using the generalized dual map $J_{p}$.

Theorem 2.4. ([6, p. 48]) Let $1<p \leq 2$ and $X$ be a Banach space, then the following are equivalent to each other.

i) $X$ is $p$-uniformly smooth;

ii) There is a constant $c>0$ satisfies that for all $x, y \in X$,

$$
\|x+y\|^{p} \leq\|x\|^{p}+p\left\langle J_{p}(x), y\right\rangle+c\|y\|^{p} .
$$

Remark. From the proof of Theorem 2.4 we know that the constant $c>0$ in (2.3) can be chosen to satisfy that $c=O\left(s_{p}(X)\right)$.

At the end of this subsection, we recall the following renorming theorem, due to Pisier [25], which provides a characterization of $p$-uniformly smooth spaces by martingale inequalities.

Theorem 2.5. ([25, Theorem 3.1]) Fix $1<p \leq 2$. Then for a Banach space $X$ the following are equivalent to each other.

i) $X$ is linear isomorphic to a $p$-uniformly smooth space;

ii) There is a constant $c>0$ such that all $X$-valued $L^{p}$-martingale $f=\left(f_{n}\right)_{n=0}^{\infty}$ the following inequality holds

$$
\sup _{n \in \mathbb{N}} \mathbb{E}\left\|f_{n}\right\|^{p} \leq C^{p} \sum_{n=0}^{\infty} \mathbb{E}\left\|d_{n, f}\right\|^{p}
$$

iii) Same as ii) for all $X$-valued dyadic martingales.

\section{Azuma-type inequalities for $p$-uniformly smooth space-valued martingales}

In this section we will study Azuma-type inequalities for martingales with values in $p$-uniformly smooth space. In addition, as we will show below, the $p$-uniform smoothness of the image space is necessary for Azuma-type inequalities to hold. At the end of this section, Azuma-type inequality for self-normalized sums will also be discussed. In the rest of this paper, we will simply use $|\cdot|$ to stand for either Euclidean or Hilbert norm. 
We now briefly recall the definition of functions of "Mittag-Leffler type". For $\alpha, \beta>0$ and $z \in \mathbb{C}$, then

$$
E_{\alpha, \beta}(z)=\sum_{k=0}^{\infty} \frac{z^{k}}{\Gamma(\alpha k+\beta)}
$$

is said to be two-parameter function of "Mittag-Leffler type", which plays an important role in the fractional calculus (see [13]).

Recall that a random process $w=\left(w_{j}\right)_{j=1}^{\infty}$ is called predictable relatively to the filtration $\left(\mathscr{F}_{j}\right)_{j=0}^{\infty}$ if $w_{j}$ is $\mathscr{F}_{j-1^{-}}$ measurable for each $j \in \mathbb{N}$. We will simply call $w=\left(w_{j}\right)_{j=1}^{\infty}$ predictable if there no confusion occurs. The following theorem is a simple variant of Pinelis' Azuma inequality (see [23, Theorem 3.6]) for 2-uniformly smooth space-valued conditionally symmetric martingales. The proof presented below is elementary and is slightly simpler than that in [23]. Hilbert space-valued martingales will suffice for our needs and in this case fruitful structures of Hilbert space could made the proof easier. In particular, we could compute the differential of $|\cdot|^{2}$ directly via the parallelogram law without the approximation argument (i.e. [23, Lemma 2.2]).

Theorem 3.1. Suppose that $f=\left(f_{j}\right)_{j=0}^{\infty}$ is a $\mathcal{H}$-valued martingale (relatively to the filtration $\left.\left(\mathscr{F}_{j}\right)_{j=0}^{\infty}\right)$ such that there exists a nonnegative predictable sequence $w=\left(w_{j}\right)_{j=1}^{\infty}$ which satisfies $\left|d_{j, f}\right| \leq w_{j}$ a.s. for each $j \in \mathbb{N}$. Then, for $b \geq\left\|\sum_{j=1}^{\infty} w_{j}^{2}\right\|_{\infty}$ and every $r>0$, we have

$$
\mathbb{P}\left\{f^{*} \geq r\right\} \leq 2 \exp \left\{-\frac{r^{2}}{2 b}\right\} .
$$

Proof. For a $C^{2}$-function $u$ defined on $\mathbb{R}$, let $\phi(t)=\cosh u(t)=E_{2,1}\left(u^{2}(t)\right)$. Then,

$$
\begin{aligned}
\phi^{\prime \prime} & =\left(\sum_{k=1}^{\infty} \frac{u^{2 k-1} \cdot u^{\prime}}{\Gamma(2 k)}\right)^{\prime} \\
& =\sum_{k=1}^{\infty} \frac{(2 k-1) u^{2 k-2} \cdot\left(u^{\prime}\right)^{2}}{\Gamma(2 k)}+\sum_{k=1}^{\infty} \frac{u^{2 k-1} \cdot u^{\prime \prime}}{\Gamma(2 k)} \\
& =\sum_{k=0}^{\infty} \frac{u^{2 k} \cdot\left(u^{\prime}\right)^{2}}{\Gamma(2 k+1)}+\sum_{k=0}^{\infty} \frac{u^{2 k} \cdot u \cdot u^{\prime \prime}}{\Gamma(2 k+2)} \\
& =E_{2,1}\left(u^{2}\right)\left(u^{\prime}\right)^{2}+E_{2,2}\left(u^{2}\right) \cdot u \cdot u^{\prime \prime} \\
& \leq E_{2,1}\left(u^{2}\right) \cdot\left(u^{2}\right)^{\prime \prime}
\end{aligned}
$$

For a $\mathcal{H}$-valued martingale $f=\left(f_{j}\right)_{j=0}^{\infty}$ and $\lambda>0$, without loss of generality, suppose that $f_{0}=0$ a.s., we define $\varphi(t):=\mathbb{E}_{j-1} \cosh \left(\lambda\left|f_{j-1}+t d_{j, f}\right|\right)$. Then, by the linearity of conditional expectation and (3.1) we have

$$
\varphi^{\prime \prime}(t) \leq \lambda^{2} \mathbb{E}_{j-1}\left\{E_{2,1}\left(\left|f_{j-1}+t d_{j, f}\right|^{2}\right) \cdot\left(\left|f_{j-1}+t d_{j, f}\right|^{2}\right)^{\prime \prime}\left\langle d_{j, f}, d_{j, f}\right\rangle\right\} .
$$

By the parallelogram law it follows that $\left(|x+t y|^{2}\right)^{\prime \prime}\langle y, y\rangle=|y|^{2}$ for all $x, y \in \mathcal{H}$ and $t \in[0,1]$. Therefore, (3.2) becomes into

for all $t \in[0,1]$.

$$
\varphi^{\prime \prime}(t) \leq \lambda^{2} \mathbb{E}_{j-1}\left\{E_{2,1}\left(\left|f_{j-1}+t d_{j, f}\right|^{2}\right) \cdot\left|d_{j, f}\right|^{2}\right\}
$$

Note that $\left|d_{j, f}\right| \leq w_{j}$ and $w_{j}$ is $\mathscr{F}_{j-1}$-measurable for each $j \in \mathbb{N}$, then we have

$$
\varphi^{\prime \prime}(t) \leq\left(\lambda \cdot w_{j}\right)^{2} \cdot \varphi(t),
$$

for all $t \in[0,1]$. Since $\varphi^{\prime}(0)=0$, then solving this differential inequality (i.e. Gronwall inequality) yields that

$$
\varphi(1) \leq \exp \left\{\frac{\lambda^{2} w_{j}^{2}}{2}\right\} \varphi(0),
$$

that is, $\mathbb{E}_{j-1} \cosh \left(\lambda\left|f_{j}\right|\right) \leq \exp \left\{\frac{\lambda^{2} w_{j}^{2}}{2}\right\} \cosh \left(\lambda\left|f_{j-1}\right|\right)$ for all $j \in \mathbb{N}$. Let

$$
g_{n}=\frac{\cosh \left(\lambda\left|f_{n}\right|\right)}{\prod_{j=1}^{n} \exp \left\{\left(\lambda \cdot w_{j}\right)^{2} / 2\right\}}
$$


for all $n \in \mathbb{N}_{0}$, then $\left(g_{n}\right)_{n=0}^{\infty}$ forms a nonnegative super-martingale. Define $\sigma:(\Omega, \mathscr{F}, \mathbb{P}) \rightarrow \mathbb{N} \cup\{\infty\}$ by $\sigma=$ $\inf \left\{n \in \mathbb{N}_{0}:\left|f_{n}\right| \geq r\right\}$, then, it is clear that $\sigma$ is a stopping time. Hence, by the "Optional Sampling Theorem" (Theorem 2.1) and the Fatou's lemma, we have

$$
\int_{\{\sigma<\infty\}} g_{\sigma} d \mathbb{P} \leq \mathbb{E}\left(g_{0}\right)=1 .
$$

Combining $b \geq\left\|\sum_{j=1}^{\infty} w_{j}^{2}\right\|_{\infty}$ with $\underline{(3.5)}$, we have

$$
\mathbb{P}\left\{f^{*} \geq r\right\} \leq \frac{\exp \left\{b \lambda^{2} / 2\right\}}{\cosh (\lambda r)}
$$

for all $r>0$ and $\lambda>0$.

Therefore, the fact $\cosh (\lambda r) \geq \frac{e^{\lambda r}}{2}$ yields that

$$
\mathbb{P}\left\{f^{*} \geq r\right\} \leq \inf _{\lambda>0}\left\{2 \exp \left\{b \lambda^{2} / 2-\lambda \cdot r\right\}\right\} .
$$

Minimize (3.6), by choosing $\lambda=\frac{r}{b}>0$, we have

$$
\mathbb{P}\left\{f^{*} \geq r\right\} \leq 2 \exp \left\{-\frac{r^{2}}{2 b}\right\}
$$

The next lemma is a "martingale dimension reduction" argument, which reduces $p$-uniformly smooth space-valued martingale to an $\mathbb{R}^{2}$-valued one. The first "martingale dimension reduction" argument was provided by Kallenberg and Sztencel in their study of continuous-time Hilbert space-valued martingales. They stated that any inequality for $\mathbb{R}^{2}$-valued continuous-time martingales which only involves some basic processes of the martingales remains true for arbitrary dimension with the same values of all constants (see [14, Theorem 3.1] for the details). However, the original proof of this theorem is long and quiet difficult, while Kwapien and Woyczynski [16, Proposition 5.8.3] provided a simple proof of this theorem for discrete-time martingales. More precisely, given a Hilbert space-valued martingale $\left(f_{j}\right)_{j=0}^{\infty}$, by Kawapień and Woyczynski's construction, one could construct an $\mathbb{R}^{2}$-valued martingale $\left(N_{j}\right)_{j=0}^{\infty}$ satisfies that

$$
\left|f_{j}\right|=\left|N_{j}\right| \text { and }\left|d_{j, f}\right|=\left|d_{j, N}\right|
$$

for all $j \in \mathbb{N}$. The main idea of Kwapień and Woyczynski's construction is to define an $\mathbb{R}^{2}$-valued martingale $\left(N_{j}\right)_{j=0}^{\infty}$ inductively such that $\left\langle d_{j, f}, f_{j-1}\right\rangle=\left\langle d_{j, N}, N_{j-1}\right\rangle$ and $\left|d_{j, f}\right|=\left|d_{j, N}\right|$ for all $j \in \mathbb{N}$. Then the structure of Euclidean norm, i.e.

$$
|x+y|^{2}=|x|^{2}+2\langle x, y\rangle+\left|y^{2}\right| \forall x, y \in \mathbb{R}^{2},
$$

ensures that $\left(N_{j}\right)_{j=0}^{\infty}$ satisfies

$$
\left|N_{j}\right|^{2}=\left|N_{j-1}\right|^{2}+2\left\langle N_{j-1}, d_{j, N}\right\rangle+\left|d_{j, N}\right|^{2} \forall j \in \mathbb{N} .
$$

Then, by induction, one gets the $\mathbb{R}^{2}$-valued martingale $\left(N_{j}\right)_{j=0}^{\infty}$ as required. However, the Kwapień and Woyczynski's approach relies on orthogonality and (3.7), which are only available in Hilbert space. In their remarkable work on Markov type and threshold embeddings of metric spaces, Ding, Lee and Peres generalized the Kwapień and Woyczynski's method by providing a "martingale dimension reduction" argument for martingales with values in general $p$-uniformly smooth space. The key insight here, due to Ding, Lee and Peres, is that for martingales with values in $p$-uniformly smooth space, one can use the inequality (2.3) instead of (3.7) (which is only available for Hilbert space) in the construction. And if one could control $N_{j}-N_{j-1}$ appropriately during their construction, then the constructed $\mathbb{R}^{2}$-valued martingale $\left(N_{j}\right)_{j=0}^{\infty}$ will satisfy the desired properties (see [8, Lemma 4.2] for the details).

Our following lemma is inspired by the construction of Ding, Lee and Peres [8, Lemma 4.2] and is even simpler than that in [8] with better constants. However, our approach is only available for certain types of martingales (such as dyadic martingales, martingales with bounded differences). Before turning into the technical details we shall describe the main idea of our construction.

Given a $p$-uniformly smooth space-valued martingale $\left(f_{j}\right)_{j=0}^{\infty}$ with $f_{0}=0$ a.s. we shall put $N_{0}=0$ a.s. and construct $\left(N_{j}\right)_{j=0}^{\infty}$ inductively as follows. Suppose that $\left(N_{j}\right)_{j=0}^{n-1}$ have been constructed, we define $N_{j}$ as the sum of two orthogonal components. The first component is to ensure that $2\left\langle N_{j-1}, N_{j}-N_{j-1}\right\rangle$ "roughly" equals to $p\left\langle J_{p}\left(f_{j-1}\right), f_{j}-f_{j-1}\right\rangle$ 
(see (3.9) below). However, by some intrinsic differences between $p$-uniformly smooth spaces and Hilbert space, these two terms as above could not equal to each other in general. Hence, an appropriate truncation will be used to make sure that the constructed $N_{j}$ "under controlled". And in order to compensate the information lost by truncation, we shall put the lost information to the second component (the orthogonal part of the first component). Finally, to make the constructed sequence $\left(N_{j}\right)_{j=0}^{\infty}$ still a martingale, we shall enlarge the filtration and attach a random sign in an appropriate way.

To organize our proof better, for $x=\left(x_{1}, x_{2}\right) \in \mathbb{R}^{2}$, we define $x^{\perp}=\left(x_{1}^{\perp}, x_{2}^{\perp}\right)$ as follows.

$$
\left(x_{1}^{\perp}, x_{2}^{\perp}\right)= \begin{cases}\left(-x_{2}, x_{1}\right) & \text { if } x \neq 0, \\ (1,0), & \text { otherwise. }\end{cases}
$$

It is clear that $\left\langle x, x^{\perp}\right\rangle=0$ for all $x \in \mathbb{R}^{2}$.

Lemma 3.2. Let $f=\left(f_{j}\right)_{j=0}^{\infty}$ be a martingale (relatively to the filtration $\left.\left(\mathscr{F}_{j}\right)_{j=0}^{\infty}\right)$ with values in $p$-uniformly smooth space $X(1<p \leq 2)$ such that there exists a nonnegative predictable sequence $w=\left(w_{j}\right)_{j=1}^{\infty}$ with $\left\|d_{j, f}\right\| \leq w_{j}$ a.s. for all $j \in \mathbb{N}$. Then, there exists an $\mathbb{R}^{2}$-valued martingale $\left(N_{j}\right)_{j=0}^{\infty}$ (relatively to another filtration) such that for all $j \in \mathbb{N}$,

i) $\left\|f_{j}-f_{0}\right\|^{p} \leq\left|N_{j}-N_{0}\right|^{2}$

ii) $\left|N_{j}-N_{j-1}\right|^{2} \leq K w_{j}^{p}$,

where $K=O\left(s_{p}(X)\right)$.

Proof. Let $\left(\varepsilon_{j}\right)_{j=1}^{\infty}$ be a sequence of independent Rademacher random variables and enlarge $\mathscr{F}_{j}$ by $\mathscr{F}_{j}^{\prime}=$ $\sigma\left(\mathscr{F}_{j}, \varepsilon_{1}, \cdots, \varepsilon_{j}\right)$, i.e. the $\sigma$-algebra generated by $\mathscr{F}_{j}$ and Rademacher random variables $\left\{\varepsilon_{1}, \cdots, \varepsilon_{j}\right\}$ for all $j \in \mathbb{N}$. Then, it is clear that $\left(f_{j}\right)_{j=0}^{\infty}$ is still a martingale and $\left(w_{j}\right)_{j=1}^{\infty}$ is still predictable relatively to the new filtration $\left(\mathscr{F}_{j}^{\prime}\right)_{j=0}^{\infty}$.

We now construct the martingale $\left(N_{j}\right)_{j=0}^{\infty}$ inductively as follows. Without loss of generality we assume that $f_{0}=0$ a.s. and put $N_{0}:=0$ a.s.. Suppose that $\left(N_{n}\right)_{n=0}^{j-1}$ have been constructed, then, let $A_{j-1}=\left\{w_{j}^{p} \leq\left|N_{j-1}\right|^{2}\right\}$ (a suitable truncation, which in order to control the constructed martingale appropriately, see (3.9), (3.13) and (3.14) below) and define

$$
\begin{aligned}
N_{j}-N_{j-1} & =\frac{p\left\langle J_{p}\left(f_{j-1}\right), f_{j}-f_{j-1}\right\rangle \cdot \mathbb{1}_{A_{j-1}}}{2} \cdot \frac{N_{j-1}}{\left|N_{j-1}\right|^{2}+\mathbb{1}_{\left\{N_{j-1}=0\right\}}} \\
& +\sqrt{c+p} \cdot \varepsilon_{j} w_{j}^{\frac{p}{2}} \cdot \frac{N_{j-1}^{\perp}}{\left|N_{j-1}\right|+\mathbb{1}_{\left\{N_{j-1}=0\right\}}} .
\end{aligned}
$$

Since $\left\langle N_{j-1}, N_{j-1}^{\perp}\right\rangle=0$ and $A_{j-1}=\left\{w_{j}^{p} \leq\left|N_{j-1}\right|^{2}\right\}$, then, as we expect,

$$
2\left\langle N_{j-1}, N_{j}-N_{j-1}\right\rangle=p\left\langle J_{p}\left(f_{j-1}\right), f_{j}-f_{j-1}\right\rangle \cdot \mathbb{1}_{A_{j-1}}
$$

Firstly, we verify that $\left(N_{j}\right)_{j=0}^{\infty}$ is a martingale relatively to the filtration $\left(\mathscr{F}_{j}^{\prime}\right)_{j=0}^{\infty}$. Indeed, note here that $w_{j}$ is $\mathscr{F}_{j-1}^{\prime}$ measurable and $\varepsilon_{j}$ is independent of $\mathscr{F}_{j-1}^{\prime}$. Then, by the linearity of conditional expectation, we have

$$
\begin{aligned}
\mathbb{E}\left(N_{j}-N_{j-1} \mid \mathscr{F}_{j-1}^{\prime}\right) & =\frac{p}{2} N_{j-1} \cdot \mathbb{E}\left(\frac{\left\langle J_{p}\left(f_{j-1}\right), f_{j}-f_{j-1}\right\rangle \cdot \mathbb{1}_{A_{j-1}}}{\left|N_{j-1}\right|^{2}+\mathbb{F}_{\left\{N_{j-1}=0\right\}}}\right) \\
& +\sqrt{c+p} \cdot w_{j}^{\frac{p}{2}} \mathbb{E}\left(\varepsilon_{j}\right) \cdot \frac{N_{j-1}^{\perp}}{\left|N_{j-1}\right|+\mathbb{1}_{\left\{N_{j-1}=0\right\}}} \\
& =\frac{p}{2} N_{j-1} \cdot \frac{\left\langle J_{p}\left(f_{j-1}\right), \mathbb{E}\left(f_{j}-f_{j-1} \mid \mathscr{F}_{j-1}^{\prime}\right)\right\rangle \cdot \mathbb{1}_{A_{j-1}}}{\left|N_{j-1}\right|^{2}+\mathbb{1}_{\left\{N_{j-1}=0\right\}}} \\
& =0
\end{aligned}
$$

for all $j \in \mathbb{N}$, which yields that $\left(N_{j}\right)_{j=0}^{\infty}$ is a martingale relatively to the filtration $\left(\mathscr{F}_{j}^{\prime}\right)_{j=0}^{\infty}$. 
Secondly, we shall verify that the $\mathbb{R}^{2}$-valued martingale $\left(N_{j}\right)_{j=0}^{\infty}$ satisfies the desired properties $\left.i\right)$ and $\left.i i\right)$. In order to do this the proof will be divided into two cases.

Case I. On the event $\left\{\omega \in \Omega: N_{j-1}(\omega) \neq 0\right\}$.

Since $X$ is a $p$-uniformly smooth space, then, Theorem 2.4 yields that for all $j \in \mathbb{N}$

$$
\begin{aligned}
\left\|f_{j}\right\|^{p} & \leq\left\|f_{j-1}\right\|^{p}+p\left\langle J_{p}\left(f_{j-1}\right), f_{j}-f_{j-1}\right\rangle+c\left\|d_{j, f}\right\|^{p} \\
& \leq\left\|f_{j-1}\right\|^{p}+p\left\langle J_{p}\left(f_{j-1}\right), f_{j}-f_{j-1}\right\rangle+c w_{j}^{p},
\end{aligned}
$$

where $c=O\left(s_{p}(X)\right)$.

Suppose that $i$ ) holds true for all $k=0, \cdots, j-1$, then (3.10) implies that

$$
\left\|f_{j}\right\|^{p} \leq\left|N_{j-1}\right|^{2}+p\left\langle J_{p}\left(f_{j-1}\right), f_{j}-f_{j-1}\right\rangle+c w_{j}^{p},
$$

with $c=O\left(s_{p}(X)\right)$.

On the other hand, since $\left\langle N_{j-1}, N_{j-1}^{\perp}\right\rangle=0$, we have

$$
\begin{aligned}
\left|N_{j}\right|^{2} & =\left|N_{j-1}\right|^{2}\left(1+\frac{p}{2} \cdot \frac{\left\langle J_{p}\left(f_{j-1}\right), f_{j}-f_{j-1}\right\rangle \cdot \mathbb{1}_{A_{j-1}}}{\left|N_{j-1}\right|^{2}}+(c+p) w_{j}^{p}\right. \\
& \geq\left|N_{j-1}\right|^{2}\left(1+p \cdot \frac{\left\langle J_{p}\left(f_{j-1}\right), f_{j}-f_{j-1}\right\rangle \cdot \mathbb{1}_{A_{j-1}}}{\left|N_{j-1}\right|^{2}}\right)+(c+p) w_{j}^{p} \\
& =\left|N_{j-1}\right|^{2}+p \cdot\left\langle J_{p}\left(f_{j-1}\right), f_{j}-f_{j-1}\right\rangle \cdot \mathbb{1}_{A_{j-1}}+(c+p) w_{j}^{p} .
\end{aligned}
$$

Then, it now suffices to estimate the term $p\left\langle J_{p}\left(f_{j-1}\right), f_{j}-f_{j-1}\right\rangle \cdot \mathbb{1}_{A_{j-1}^{c}}$, where $A_{j-1}^{c}$ is the complementary set of $A_{j-1}$. Note that $A_{j-1}^{c}=\left\{w_{j}^{p}>\left|N_{j-1}\right|^{2}\right\}$, we have

$$
\begin{aligned}
p\left\langle J_{p}\left(f_{j-1}\right), f_{j}-f_{j-1}\right\rangle \cdot \mathbb{1}_{A_{j-1}^{c}} & \leq p\left\|f_{j-1}\right\|^{p-1}\left\|f_{j}-f_{j-1}\right\| \cdot \mathbb{1}_{A_{j-1}^{c}} \\
& \leq p\left|N_{j-1}\right|^{\frac{2(p-1)}{p}}\left\|f_{j}-f_{j-1}\right\| \cdot \mathbb{1}_{A_{j-1}^{c}} \\
& \leq p w_{j}^{p},
\end{aligned}
$$

Then, combining with (3.11), (3.12) and (3.13) it follows that $\left\|f_{j}\right\|^{p} \leq\left|N_{j}\right|^{2}$, which completes our proof of $i$ ).

To prove that $\left(N_{j}\right)_{j=0}^{\infty}$ satisfies $\left.i i\right)$, we note here that $\left\langle N_{j-1}, N_{j-1}^{\perp}\right\rangle=0$, then,

$$
\left|N_{j}-N_{j-1}\right|^{2}=\frac{p^{2}}{4} \cdot \frac{\left\langle J_{p}\left(f_{j-1}\right), f_{j}-f_{j-1}\right\rangle^{2} \cdot \mathbb{1}_{A_{j-1}}}{\left|N_{j-1}\right|^{2}}+(c+p) w_{j}^{p} .
$$

By $i$ ), it follows that

$$
\begin{aligned}
\left|N_{j}-N_{j-1}\right|^{2} & \leq \frac{p^{2}}{4} \cdot \frac{\left\|f_{j-1}\right\|^{2(p-1)}\left\|f_{j}-f_{j-1}\right\|^{2} \cdot \mathbb{1}_{A_{j-1}}}{\left|N_{j-1}\right|^{2}}+(c+p) w_{j}^{p} \\
& \leq \frac{p^{2}}{4} \cdot \frac{\left|N_{j-1}\right|^{\frac{4(p-1)}{p}} w_{j}^{2} \cdot \mathbb{1}_{A_{j-1}}}{\left|N_{j-1}\right|^{2}}+(c+p) w_{j}^{p} \\
& =\frac{p^{2}}{4} \cdot \frac{w_{j}^{2} \cdot \mathbb{1}_{A_{j-1}}}{\left|N_{j-1}\right|^{\frac{2(2-p)}{p}}}+(c+p) w_{j}^{p},
\end{aligned}
$$

Note that $1<p \leq 2$ and $A_{j-1}=\left\{w_{j}^{p} \leq\left|N_{j-1}\right|^{2}\right\}$, then $\frac{4-2 p}{p} \geq 0$ and (3.14) can be further estimated as follows

$$
\begin{aligned}
\left|N_{j}-N_{j-1}\right|^{2} & \leq \frac{p^{2}}{4} \cdot \frac{w_{j}^{2}}{w_{j}^{2-p}}+(c+p) w_{j}^{p} \\
& =\left(\frac{p^{2}}{4}+c+p\right) w_{j}^{p}
\end{aligned}
$$

Denote that $K=\frac{p^{2}}{4}+c+p \leq c+3$, then

$$
\left|N_{j}-N_{j-1}\right|^{2} \leq K w_{j}^{p}, \forall j \in \mathbb{N}
$$


with $K=O\left(s_{p}(X)\right)$.

Case II. On the event $\left\{\omega \in \Omega: N_{j-1}(\omega)=0\right\}$, it is easy to verify that the martingale $\left(N_{j}\right)_{j=0}^{\infty}$ satisfies the desired properties $i$ ) and $i i$ ).

Indeed, by induction, we have $\left\|f_{j-1}-f_{0}\right\|^{p} \leq\left|N_{j-1}-N_{0}\right|^{2}$, which yields that $f_{j-1}=0$ on the event $\{\omega \in \Omega$ : $\left.N_{j-1}(\omega)=0\right\}$. Hence,

$$
\left|N_{j}-N_{j-1}\right|^{2}=\left|N_{j}\right|^{2}=(c+p) w_{j}^{p} \geq(c+p)\left\|f_{j}-f_{j-1}\right\|^{p} \geq\left\|f_{j}\right\|^{p},
$$

which completes our proof.

With the help of the Lemma 3.2 we can now prove the following Azuma-type inequality for $p$-uniformly smooth space-valued martingales.

Theorem 3.3. Let $f=\left(f_{j}\right)_{j=0}^{\infty}$ be a martingale with values in $p$-uniformly smooth space $(1<p \leq 2)$ such that there exists a non-negative predictable sequence $w=\left(w_{j}\right)_{j=1}^{\infty}$ with $\left\|d_{j, f}\right\| \leq w_{j}$ for all $j \in \mathbb{N}$. Then, there exists a constant $K$ depending only on $X$ such that for all $r \geq 0$,

$$
\mathbb{P}\left\{f^{*} \geq r\right\} \leq 2 \exp \left\{-\frac{r^{p}}{2 K\left\|\sum_{j=1}^{\infty} w_{j}^{p}\right\|_{\infty}}\right\},
$$

where $K=O\left(s_{p}(X)\right)$.

Proof. Since $w=\left(w_{j}\right)_{j=0}^{\infty}$ is a predictable sequence, then apply Lemma 3.2, there exists an $\mathbb{R}^{2}$-valued martingale $N=\left(N_{j}\right)_{j=0}^{\infty}$ (relative another filtration) satisfying

i) $\left\|f_{j}\right\|^{p} \leq\left|N_{j}\right|^{2}$

ii) $\left|N_{j}-N_{j-1}\right|^{2} \leq K w_{j}^{p}$,

for all $j \in \mathbb{N}$, where $K=O\left(s_{p}(X)\right)$.

By Theorem 3.1, we have for all $r \geq 0$,

$$
\mathbb{P}\left\{N^{*} \geq r\right\} \leq 2 \exp \left\{-\frac{r^{2}}{2 K\left\|\sum_{j=1}^{\infty} w_{j}^{p}\right\|_{\infty}}\right\} .
$$

By $i$ ) we have $\left\{\left\|f_{n}\right\| \geq t^{\frac{2}{p}}\right\} \subseteq\left\{\left|N_{n}\right|^{2} \geq t^{2}\right\}=\left\{\left|N_{n}\right| \geq t\right\}$ for all $n \in \mathbb{N}$ and $t>0$. Therefore, for $r>0$, we have $\left\{\left\|f_{n}\right\| \geq r\right\} \subseteq\left\{\left\|N_{n}\right\| \geq r^{\frac{p}{2}}\right\}$ and hence, combining with (3.15), it follows that

$$
\mathbb{P}\left\{f^{*} \geq r\right\} \leq 2 \exp \left\{-\frac{r^{p}}{2 K\left\|\sum_{j=1}^{\infty} w_{j}^{p}\right\|_{\infty}}\right\} .
$$

This completes our proof.

Remark. For the case $\left\|\sum_{j=1}^{\infty} w_{j}^{p}\right\|_{\infty}=\infty$ the inequality 3.17 holds true trivially.

The following two inequalities are easy corollaries of Theorem 3.3 The first one is a generalization of Pinelis' inequality for 2-uniformly smooth space-valued martingales [22] and the second one can be viewed as an improvement of Naor's Azuma inequality.

Corollary 3.4. Suppose that $f=\left(f_{j}\right)_{j=0}^{\infty}$ is a conditionally symmetric martingale with values in a $p$-uniformly smooth space $X(1<p \leq 2)$. If $b$ is such that $b \geq\left\|S_{p}^{p}(f)\right\|_{\infty}$, then the following inequality holds for all $r \geq 0$,

$$
\mathbb{P}\left\{f^{*} \geq r\right\} \leq 2 \exp \left\{-\frac{r^{p}}{2 K b}\right\}
$$

where $K$ depends only on $X$. 
Proof. Since $f=\left(f_{j}\right)_{j=0}^{\infty}$ is a conditionally symmetric martingale with valued in a $p$-uniformly smooth space $X$, then, by Proposition 2.2, there exists a filtration $\left(\mathscr{G}_{j}\right)_{j=0}^{\infty}$ such that $f=\left(f_{j}\right)_{j=0}^{\infty}$ is a martingale and $\left(\left\|d_{j, f}\right\|\right)_{j=1}^{\infty}$ is predictable relatively to the filtration $\left(\mathscr{G}_{j}\right)_{j=0}^{\infty}$.

Then, by Theorem 3.3. we have for all $r \geq 0$,

$$
\mathbb{P}\left\{f^{*} \geq r\right\} \leq 2 \exp \left\{-\frac{r^{p}}{2 K b}\right\}
$$

where $K=O\left(s_{p}(X)\right)$. This completes our proof.

Corollary 3.5. Let $f=\left(f_{j}\right)_{j=0}^{\infty}$ be martingale with values in $p$-uniformly smooth Banach space $X(1<p \leq 2)$. If $b$ is such that $b \geq \sum_{j=1}^{\infty}\left\|d_{j, f}\right\|_{\infty}^{p}$, then the following inequality holds for all $r \geq 0$,

$$
\mathbb{P}\left\{f^{*} \geq r\right\} \leq 2 \exp \left\{-\frac{r^{p}}{2 K b}\right\}
$$

where $K$ depends only on $X$.

Proof. Obviously $\left(\left\|d_{j, f}\right\|_{\infty}\right)_{j=0}^{\infty}$ is a predictable sequence such that $\left\|d_{j, f}\right\| \leq\left\|d_{j, f}\right\|_{\infty}$ for each $j \in \mathbb{N}$.

Theorem 3.3 states that certain type of Azuma inequality holds for martingales with values in $p$-uniformly smooth space and it is natural to consider the inverse question: Is the $p$-uniform smoothness of the image space necessary for Azuma-type inequalities to hold? We shall answer this question affirmatively in the following. Before doing this we now introduce the definition of "Azuma type" for Banach spaces.

Definition 3.1. A Banach space $X$ is said to have Azuma type $p$, if there exists a constant $K>0$ depending on $X$ such that for every martingale $f=\left(f_{j}\right)_{j=0}^{\infty}$ with values in $X$ and every predictable sequence $w=\left(w_{j}\right)_{j=1}^{\infty}$ with $\left\|d_{j, f}\right\| \leq w_{j}$ for all $j \in \mathbb{N}$, then the following inequality holds, for $r>0$,

$$
\mathbb{P}\left\{f^{*} \geq r\right\} \leq 2 \exp \left\{-\frac{r^{p}}{K\left\|\sum_{j=1}^{\infty} w_{j}^{p}\right\|_{\infty}}\right\} .
$$

The smallest constant $K$ such that inequality (3.17) holds is called the Azuma type constant of $X$ denoted by $K_{p, X}$.

Remark. The constant "2" that appears in the definition of "Azuma type" does not have any special meaning, which can be replaced by any other absolute positive constants.

In other word, the Theorem 3.3 asserts that $p$-uniformly smooth spaces are of Azuma type $p$. We now at the position to prove that a Banach space $X$ is of Azuma type $p$ then it must be $p$-uniformly smooth, up to a linear isomorphism. Our proof of this statement is based on a "good- $\lambda$ inequality" of Burkholder [4].

Lemma 3.6. Let $X$ be a Banach space with Azuma type $p$ with constant $K_{p, X}$, then for every conditionally symmetric martingale $f=\left(f_{j}\right)_{j=0}^{\infty}$ (relatively to the filtration $\left.\left(\mathscr{F}_{j}\right)_{j=0}^{\infty}\right)$ the following inequality holds. For $\beta>0$ and $0<\delta<$ $\beta-1$ we have

$$
\mathbb{P}\left\{f^{*}>\beta \lambda, S_{p}(f) \leq \delta \lambda\right\} \leq 2 \exp \left\{-\frac{(\beta-1-\delta)^{p}}{K_{p, X} \delta^{p}}\right\} \mathbb{P}\left\{f^{*}>\lambda\right\}
$$

for all $\lambda>0$.

Proof. For conditionally symmetric martingale $f=\left(f_{j}\right)_{j=0}^{\infty}$, applying Proposition 2.2 , there exits a filtration $\left(\mathscr{G}_{j}\right)_{j=0}^{\infty}$ such that $\left(f_{j}\right)_{j=0}^{\infty}$ is also a martingale relatively to the filtration $\left(\mathscr{G}_{j}\right)_{j=0}^{\infty}$ and $\left\|d_{j, f}\right\|$ is $\mathscr{G}_{j-1}$-measurable for each $j \in \mathbb{N}$. Define stopping times as follows

$$
\begin{gathered}
\mu=\inf \left\{n \in \mathbb{N}_{0}:\left\|f_{n}\right\|>\lambda\right\}, \\
\nu=\inf \left\{n \in \mathbb{N}_{0}:\left\|f_{n}\right\|>\beta \lambda\right\}, \\
\sigma=\inf \left\{n \in \mathbb{N}_{0}: S_{p, n+1}(f)>\delta \lambda\right\} .
\end{gathered}
$$

Then it is clear that $\left\{f^{*}>\lambda\right\}=\{\mu<\infty\},\left\{f^{*}>\beta \lambda\right\}=\{\nu<\infty\}$ and $\left\{S_{p}(f) \leq \delta \lambda\right\}=\{\sigma=\infty\}$. Let

$$
h_{n}=\sum_{j=0}^{n} \mathbb{1}_{\{\mu<j \leq \nu \wedge \sigma\}} d_{j, f},
$$


for all $n \in \mathbb{N}$ and $h_{0}:=0$ a.s. for convenience. We now verify that $h=\left(h_{j}\right)_{j=0}^{\infty}$ is a martingale relatively to the filtration $\left(\mathscr{G}_{j}\right)_{j=0}^{\infty}$. Indeed, it suffices to show that $\{\mu<j \leq \nu \wedge \sigma\}$ is $\mathscr{G}_{j-1}$-measurable for each $j \in \mathbb{N}$. Since

$$
\begin{array}{r}
\{\mu<n\}=\bigcup_{j=0}^{n-1}\left\{\left\|f_{j}\right\|>\lambda\right\}, \\
\{n \leq \nu\}=\bigcap_{j=0}^{n-1}\left\{\left\|f_{j}\right\| \leq \beta \lambda\right\},
\end{array}
$$

and

$$
\{n \leq \sigma\}=\bigcap_{j=0}^{n}\left\{S_{p, j}(f) \leq \delta \lambda\right\},
$$

then by the fact that $\left\|d_{j, f}\right\|$ are $\mathscr{G}_{j-1}$-measurable we have $\{\mu<j \leq \nu \wedge \sigma\}$ is $\mathscr{G}_{j-1}$ measurable for each $j \in \mathbb{N}$, which entails that $h=\left(h_{j}\right)_{j=0}^{\infty}$ is a martingale with $\left\|d_{j, h}\right\|$ is $\mathscr{G}_{j-1}$-measurable for each $j \in \mathbb{N}$.

By the definition of stopping times $\mu, \nu$ and $\sigma$, we have

$$
\left\{f^{*}>\beta \lambda, S_{p}(f) \leq \delta \lambda\right\}=\{\nu<\infty, \sigma=\infty\} \subseteq\left\{h^{*}>(\beta-1-\delta) \lambda\right\} .
$$

We now estimate the term $\left\|S_{p}(h)\right\|_{\infty}$. It is clear that $h=0$ a.s. on $\{\mu=\infty\}$, then, by the definition of $h=\left(h_{j}\right)_{j=0}^{\infty}$ and $\sigma$ we have $S_{p}(h) \leq S_{p, \sigma}(f) \leq \delta \lambda$ on $\{\mu<\infty\}$ almost surely.

Hence, by the fact that $X$ is of Azuma type $p$ with Azum type constant $K_{p, X}$, then the following inequality holds

$$
\begin{aligned}
\mathbb{P}\left\{f^{*}>\beta \lambda, S_{p}(f) \leq \delta \lambda\right\} & \leq \mathbb{P}\left\{h^{*}>(\beta-1-\delta) \lambda\right\} \\
& =\int_{\{\mu<\infty\}} \mathbb{1}_{\left\{h^{*}>(\beta-1-\delta) \lambda\right\}} d \mathbb{P} \\
& =\int \mathbb{E}\left(\mathbb{1}_{\left\{h^{*}>(\beta-1-\delta) \lambda\right\}} \cdot \mathbb{1}_{\{\mu<\infty\}} \mid \mathscr{G}_{\mu}\right) d \mathbb{P} \\
& =\mathbb{P}\left\{h^{*}>(\beta-1-\delta) \lambda\right\} \cdot \mathbb{P}\{\mu<\infty\} \\
& \leq 2 \exp \left\{-\frac{(\beta-1-\delta)^{p}}{K_{p, X} \delta^{p}}\right\} \mathbb{P}\left\{f^{*}>\lambda\right\} .
\end{aligned}
$$

Combining with the "good- $\lambda$ inequality" above and the renorming theorem of Pisier [25] (corresponding to Theorem 2.5), we can now assert that a Banach space which is of Azuma type $p$ must be linear isomorphic to a $p$-uniformly smooth space.

Theorem 3.7. Suppose that $X$ is a Banach space with Azuma type $p(1<p \leq 2)$. Then, $X$ is linear isomorphic to a $p$-uniformly smooth space.

Proof. Suppose that $X$ has the Azuma type $p$ with Azuma type constant $K_{p, X}$. Then, by Lemma 3.6 for every $L^{p}$ dyadic martingale $f=\left(f_{j}\right)_{j=0}^{\infty}$ with values in $X$, we have, for all $\lambda>0$,

$$
\mathbb{P}\left\{f^{*}>\beta \lambda, S_{p}(f) \leq \delta \lambda\right\} \leq 2 \exp \left\{-\frac{(\beta-1-\delta)^{p}}{K_{p, X} \delta^{p}}\right\} \mathbb{P}\left\{f^{*}>\lambda\right\} .
$$

Choose $\beta=2$ and $\delta=\frac{1}{1+\left((r+2) \log 2 K_{p, X}\right)^{1 / p}}$, then, [4, Lemma 7.1] yields that

$$
\left\|f^{*}\right\|_{r}^{r} \leq 4^{r+1}\left((r+2) \log 2 \cdot K_{p, X}\right)^{\frac{r}{p}}\left\|S_{p}(f)\right\|_{r}^{r}, 1 \leq r<\infty .
$$

By the Pisier's renorming theorem (see Theorem 2.5), it follows that $X$ is linear isomorphic to a $p$-uniformly smooth space.

Remark. Combining with Theorem 3.3 and Theorem 3.7, we obtain a characterization of $p$-uniformly smooth spaces in terms of Azuma-type inequalities. 
We conclude this section by providing a further refinement of the Azuma-type inequality for Banach space-valued martingales. Observe that if $f=\left(f_{j}\right)_{j=0}^{\infty}$ is a conditionally symmetric martingales with values in $p$-uniformly smooth space, then the Corollary 3.4 yields that, for all $r \geq 0$,

$$
\mathbb{P}\left\{\left\|f_{n}-f_{0}\right\| \geq r\right\} \leq 2 \exp \left\{-\frac{r^{p}}{2 K\left\|S_{p, n}(f)\right\|_{\infty}^{p}}\right\}
$$

where $K=O\left(s_{p}(X)\right)$. Equivalently, (3.18) can be restated as follows

$$
\mathbb{P}\left\{\frac{\left\|f_{n}-f_{0}\right\|}{\left\|S_{p, n}(f)\right\|_{\infty}} \geq r\right\} \leq 2 \exp \left\{-\frac{r^{p}}{2 K}\right\}
$$

Note that $\left\{\frac{\left\|f_{n}-f_{0}\right\|}{\left\|S_{p, n}(f)\right\|_{\infty}} \geq r\right\} \subseteq\left\{\frac{\left\|f_{n}-f_{0}\right\|}{S_{p, n}(f)} \geq r\right\}$ for all $r \geq 0$, hence, the following Azuma-type inequality for self-normalized sums is an improvement of the classical Azuma inequality for conditionally symmetric martingales.

Theorem 3.8. Let $f=\left(f_{n}\right)_{n=0}^{\infty}$ be a conditionally symmetric martingale with values in $p$-uniformly smooth Banach space. Then, for every $r \geq 0$, the following inequality holds

$$
\mathbb{P}\left\{\frac{\left\|f_{n}-f_{0}\right\|}{S_{p, n}(f)} \geq r\right\} \leq 4 \exp \left\{-\frac{r^{p}}{2 K}\right\}
$$

for all $n \in \mathbb{N}$, where $K=O\left(s_{p}(X)\right)$.

Proof. We begin our proof with a special case, namely Hilbert space-valued martingales such that the norm of martingale differences dominated by non-negative predictable sequence. And then we conclude our proof by applying the "martingale dimension reduction" lemma to reduce the case from $p$-uniformly smooth space-valued to the case of Hilbert space-valued. Without loss of generality we assume martingale $\left(f_{j}\right)_{j=0}^{\infty}$ satisfies $f_{0}=0$ a.s..

Step I. Suppose that $f=\left(f_{j}\right)_{j=0}^{\infty}$ be a Hilbert space-valued martingale such that there exists a predictable sequence $w=\left(w_{j}\right)_{j=1}^{\infty}$ with $\left|d_{j, f}\right| \leq w_{j}$ for all $j \in \mathbb{N}$ and denote that $S_{2, n}(w)=\left(\sum_{j=1}^{n} w_{j}^{2}\right)^{1 / 2}$ for all $n \in \mathbb{N}$.

Let $B=\left\{\frac{\left|f_{n}\right|}{S_{2, n}(w)} \geq r\right\}$ for $r>0$, then by the fact that $e^{t} \leq 2 \cosh (t) \leq 2 e^{t}$ for all $t \in \mathbb{R}$, and the Cauchy-Schwarz inequality, we have the following

$$
\begin{aligned}
\mathbb{P}(B) & \leq 2 \inf _{\lambda>0} \int \frac{\cosh \frac{\lambda}{2}\left|f_{n}\right|}{\exp \left\{\frac{\lambda r}{2} S_{2, n}(w)\right\}} \cdot \mathbb{1}_{B} d \mathbb{P} \\
& =2 \inf _{\lambda>0} \int \frac{\cosh \frac{\lambda}{2}\left|f_{n}\right|}{\exp \left\{\frac{\lambda^{2}}{4} S_{2, n}^{2}(w)\right\}} \cdot \exp \left\{\frac{\lambda^{2}}{4} S_{2, n}^{2}(w)-\frac{\lambda r}{2} S_{2, n}(w)\right\} \cdot \mathbb{1}_{B} d \mathbb{P} \\
& \leq 2 \inf _{\lambda>0}\left(\int \frac{\cosh \lambda\left|f_{n}\right|}{\exp \left\{\frac{\lambda^{2}}{2} S_{2, n}^{2}(w)\right\}} d \mathbb{P}\right)^{1 / 2} \cdot\left(\int \exp \left\{\frac{\lambda^{2}}{2} S_{2, n}^{2}(w)-\lambda r S_{2, n}(w)\right\} \cdot \mathbb{1}_{B} d \mathbb{P}\right)^{1 / 2} .
\end{aligned}
$$

By the proof of Theorem 3.1 we know that $\left\{\frac{\cosh \lambda\left|f_{n}\right|}{\exp \left\{\frac{\lambda^{2}}{2} S_{2, n}^{2}(w)\right\}}\right\}_{n=0}^{\infty}$ forms a super-martingale. Then, (3.20) becomes into

$$
\begin{aligned}
\mathbb{P}(B) & \leq 2 \inf _{\lambda>0}\left(\int \exp \left\{\frac{\lambda^{2}}{2} S_{2, n}^{2}(w)-\lambda r S_{2, n}(w)\right\} \cdot \mathbb{1}_{B} d \mathbb{P}\right)^{1 / 2} \\
& =2 \int\left(\exp \left\{-\frac{r^{2}}{2}\right\} \cdot \mathbb{1}_{B} d \mathbb{P}\right)^{1 / 2} \\
& =2 \exp \left\{-\frac{r^{2}}{4}\right\} \cdot \mathbb{P}(B)^{1 / 2} .
\end{aligned}
$$

Therefore,

$$
\mathbb{P}\left\{\frac{\left|f_{n}\right|}{S_{2, n}(w)} \geq r\right\}=\mathbb{P}(B) \leq 4 \exp \left\{-\frac{r^{2}}{2}\right\}
$$


for all $n \in \mathbb{N}$.

Step II. Suppose that $f=\left(f_{j}\right)_{j=0}^{\infty}$ is a conditionally symmetric martingale with values in $p$-uniformly smooth space, then there exists another filtration $\left(\mathscr{G}_{j}\right)_{j=0}^{\infty}$ such that $f=\left(f_{j}\right)_{j=0}^{\infty}$ is a martingale relatively to the filtration $\mathscr{G}$ and $\left\|d_{j, f}\right\|$ is $\mathscr{G}_{j-1}$-measurable for each $j \in \mathbb{N}$. By Lemma 3.2, there exists an $\mathbb{R}^{2}$-valued martingale $\left\{N_{j}\right\}_{j=0}^{\infty}$ such that for all $j \in \mathbb{N}$

i) $\left\|f_{j}\right\|^{p} \leq\left|N_{j}\right|^{2}$,

ii) $\left|N_{j}-N_{j-1}\right|^{2} \leq K\left\|f_{j}-f_{j-1}\right\|^{p}$,

where $K=O\left(s_{p}(X)\right)$.

Then, applying (3.21) we have the following

$$
\begin{aligned}
\mathbb{P}\left\{\frac{\left\|f_{n}\right\|}{S_{p, n}(f)} \geq r\right\} & =\mathbb{P}\left\{\frac{\left\|f_{n}\right\|^{p}}{S_{p, n}^{p}(f)} \geq r^{p}\right\} \\
& \leq \mathbb{P}\left\{\frac{\left|N_{n}\right|^{2}}{S_{2, n}^{2}\left(N_{n}\right)} \geq \frac{r^{p}}{K}\right\} \\
& \leq 4 \exp \left\{-\frac{r^{p}}{2 K}\right\},
\end{aligned}
$$

for all $n \in \mathbb{N}$, which completes our proof.

Remark. The constant " 4 " of (3.19) is not the best possible and it will be "2" for real-valued conditionally symmetric martingales. However, if we ignore the universal constant here, the inequality (3.19) indeed provides the right degree of concentration.

\section{Some further inequalities for Banach space-valued martingales}

In this section, methods developed in the previous section will be used to derive some further inequalities for Banach space-valued martingales, such as moment inequalities for double dyadic martingales and De la Peña-type inequality for conditionally symmetric martingales.

\subsection{Moment inequalities for Banach space-valued double dyadic martingales}

Moment inequalities for double dyadic martingales are important to Harmonic analysis. Pipher [24] provided a moment inequality for double dyadic martingales (see the definition below) and then applied it to the study the exponential square integrability of $\left|f-f_{Q}\right|$ over $Q$ in the bidisc case of two parameter kernel. And Bañuelos [3] extended Phipher's results to continuous-time martingales on Brownian filtration and then applied them to the study of Riesz transforms.

In the rest of this subsection we will apply the methods developed in Section 3 to deduce moment inequalities for Banach space-valued double dyadic martingales. Recall that a random process $f=\left(f_{n}\right)_{n=0}^{\infty}$ is said to be dyadic martingale (or, Paley-Walsh martingale) if $f=\left(f_{n}\right)_{n=0}^{\infty}$ is martingale relatively to the filtration $\left(\mathscr{F}_{n}\right)_{n=0}^{\infty}$, where $\mathscr{F}_{n}$ are $\sigma$-sub-algebra generated by the dyadic intervals of length $2^{-n}$ in $[0,1]$. A double dyadic martingale is a double indexed random process $\left(f_{n}^{j}\right)_{j, n}$ such that $\left(f_{n}^{j}\right)_{j, n}$ is a dyadic martingale in $j$ for each fixed $n$ and also a dyadic martingale in $n$ for each fixed $j$. The following inequalities can be viewed as a variant of [3, Theorem 1.1] for Banach space-valued double dyadic martingales.

Theorem 4.1. Suppose that $f^{j}=\left(f_{n}^{j}\right)_{n=0}^{\infty}$ are dyadic martingales with values in $p$-uniformly smooth (resp. $q$ uniformly convex) Banach space for $1<p \leq 2$ (resp. $2 \leq q<\infty), j=1,2, \cdots, m$. Then, there exists constant $K$ depending only on $X$ such that following inequalities hold

$$
\begin{gathered}
\left\|\left(\sum_{j=1}^{m}\left\|f_{n}^{j}\right\|^{p}\right)^{1 / p}\right\|_{r} \leq K r^{1 / p}\left\|\left(\sum_{j=1}^{m} S_{p, n}^{p}\left(f^{j}\right)\right)^{1 / p}\right\|_{r}, \\
\text { resp. } \left.\left\|\left(\sum_{j=1}^{m} S_{q, n}^{q}\left(f^{j}\right)\right)^{1 / q}\right\|_{r} \leq K r^{1 / q}\left\|\left(\sum_{j=1}^{m}\left\|f_{n}^{j}\right\|^{q}\right)^{1 / q}\right\|_{r},\right)
\end{gathered}
$$

for all $2 \leq r<\infty$ and $n \in \mathbb{N}$. 
To prove the theorem we will first deal with Hilbert space-valued dyadic martingales and then apply the "martingale dimension reduction" argument to reduce martingales from $p$-uniformly smooth space-valued to $\mathbb{R}^{2}$-valued. The following lemma is a variant of Theorem 3.1

Lemma 4.2. Suppose that $f^{j}=\left(f_{n}^{j}\right)_{n=0}^{\infty}$ are dyadic martingales with values in Hilbert space $\mathcal{H}$, for $j=1,2, \cdots, m$. Then, for every $r \geq 0$

$$
\mathbb{P}\left\{\left(\sum_{j=1}^{m}\left|f_{n}^{j}\right|^{2}\right)^{1 / 2} \geq r\right\} \leq 2 \exp \left\{-\frac{r^{2}}{2\left\|\sum_{j=1}^{m} S_{2, n}^{2}\left(f^{j}\right)\right\|_{\infty}}\right\}
$$

for all $n \in \mathbb{N}$.

Proof. Let $u(t)=\left(\sum_{j=1}^{m}\left|f_{n-1}^{j}+t d_{n, f^{j}}\right|^{2}\right)^{1 / 2}$ and $\varphi(t)=\mathbb{E}_{n}(\cosh \lambda u(t))$. Note that $f^{j}=\left(f_{n}^{j}\right)_{n=0}^{\infty}$ are dyadic martingales for all $j=1, \cdots, m$, and hence $\left|d_{n, f^{j}}\right|$ is $\mathscr{F}_{n-1}$-measurable for each $n \in \mathbb{N}$ and $j=1,2 \cdots, m$.

By the proof of Theorem 3.1, we have $\varphi(1) \leq \exp \left\{\frac{\lambda^{2} \sum_{j=1}^{m}\left|d_{n, f j}\right|^{2}}{2}\right\} \varphi(0)$, then it follows that $\left(g_{n}\right)_{n=0}^{\infty}$ forms a super-martingale, where

$$
g_{n}=\frac{\cosh \lambda\left(\sum_{j=1}^{m}\left|f_{n}^{j}\right|^{2}\right)^{1 / 2}}{\prod_{j=1}^{m} \exp \left\{\frac{\lambda^{2} S_{2, n}^{2}\left(f^{j}\right)}{2}\right\}}, n \in \mathbb{N}_{0}, \lambda>0 .
$$

Consequently, the result follows from the Markov inequality and the fact that $\int g_{n} d \mathbb{P} \leq \int g_{0} d \mathbb{P}=1$.

Proof of Theorem 4.1] Step I. Suppose that $f^{j}=\left(f_{n}^{j}\right)_{n=0}^{\infty}, j=1, \cdots, m$ are dyadic martingales with values in $p$ uniformly smooth space, then, by the "martingale dimension reduction" argument (Lemma 3.2), it follows that there exists $\mathbb{R}^{2}$-valued martingales $N^{j}=\left(N_{n}^{j}\right)_{n=0}^{\infty}$ such that

$$
\left\|f_{n}^{j}\right\|^{p} \leq\left|N_{n}^{j}\right|^{2}
$$

and

$$
\left|N_{n}^{j}-N_{n-1}^{j}\right|^{2} \leq K_{1}\left\|d_{n, f^{j}}\right\|^{p}
$$

$j=1, \cdots, m$ with $K_{1}=O\left(s_{p}(X)\right)$.

Hence, analogous to the proof of Theorem 3.7 we can deduce the following inequality by using Lemma 3.6 and Lemma 4.2

$$
\left\|\left(\sum_{j=1}^{m}\left\|f_{n}^{j}\right\|^{p}\right)^{1 / 2}\right\|_{\gamma}^{\gamma} \leq\left(\gamma K_{1}\right)^{\gamma / 2}\left\|\left(\sum_{j=1}^{m} S_{p, n}^{p}\left(f^{j}\right)\right)^{1 / 2}\right\|_{\gamma}^{\gamma},
$$

for all $1 \leq \gamma<\infty$. Therefore, the following holds

$$
\int\left(\sum_{j=1}^{m}\left\|f_{n}^{j}\right\|^{p}\right)^{\delta} d \mathbb{P} \leq\left(2 \delta K_{1}\right)^{\delta} \int\left(\sum_{j=1}^{m} S_{p, n}^{p}\left(f^{j}\right)\right)^{\delta} d \mathbb{P},
$$

for $\frac{1}{2} \leq \delta=\frac{\gamma}{2}<\infty$. Note that $1<p \leq 2$, hence, by 4.5), the following inequality holds true for all $1 \leq r<\infty$,

$$
\int\left(\sum_{j=1}^{m}\left\|f_{n}^{j}\right\|^{p}\right)^{r / p} d \mathbb{P} \leq\left(\frac{2 r K_{1}}{p}\right)^{r / p} \int\left(\sum_{j=1}^{m} S_{p, n}^{p}\left(f^{j}\right)\right)^{r / p} d \mathbb{P} .
$$

Consequently

$$
\left\|\left(\sum_{j=1}^{m}\left\|f_{n}^{j}\right\|^{p}\right)^{1 / p}\right\|_{r} \leq\left(2 K_{1}\right)^{1 / p} r^{1 / p}\left\|\left(\sum_{j=1}^{m} S_{p, n}^{p}\left(f^{j}\right)\right)^{1 / p}\right\|_{r},
$$

for all $1 \leq r<\infty$. 
Step II. We prove the inequality (4.2) via duality argument. For $2 \leq q, r<\infty$, denote that $r^{\prime}=\frac{r}{r-1}$ and $q^{\prime}=\frac{q}{q-1}$, then, for any $0<\varepsilon<1$, there exists a sequence $\left(\varphi_{l, j}\right)_{j=1, l=1}^{m, n} \in L^{r^{\prime}}\left(X^{*}\right)$ such that

$$
\left\|\left(\sum_{j=1}^{m} \sum_{l=1}^{n}\left\|\varphi_{l, j}\right\|^{q^{\prime}}\right)^{1 / q^{\prime}}\right\|_{r^{\prime}} \leq 1
$$

and

$$
\begin{aligned}
(1-\varepsilon)\left\|\left(\sum_{j=1}^{m} S_{q, n}^{q}\left(f^{j}\right)\right)^{1 / q}\right\|_{r} & \leq \int \sum_{j=1}^{m} \sum_{l=1}^{n}\left\langle d_{l, f^{j}}, \varphi_{l, j}\right\rangle d \mathbb{P} \\
& =\int \sum_{j=1}^{m} \sum_{l=1}^{n}\left\langle d_{l, f^{j}},\left(\mathbb{E}_{l}-\mathbb{E}_{l-1}\right) \varphi_{l, j}\right\rangle d \mathbb{P} \\
& =\int \sum_{j=1}^{m}\left\langle f_{n}^{j}, g_{n}^{j}\right\rangle d \mathbb{P},
\end{aligned}
$$

where $g_{n}^{j}=\sum_{l=1}^{n}\left(\mathbb{E}_{l}-\mathbb{E}_{l-1}\right) \varphi_{l, j}$ for all $j=1, \cdots, m$. We now estimate the term $\int \sum_{j=1}^{m}\left\langle f_{n}^{j}, g_{n}^{j}\right\rangle d \mathbb{P}$ by a duality argument. Indeed, if $X$ is of $q$-uniformly convex then $X^{*}$ must be $q^{\prime}$-uniformly smooth and hence, by the first part of our proof and the Hölder inequality, we have

$$
\begin{aligned}
\int \sum_{j=1}^{m}\left\langle f_{n}^{j}, g_{n}^{j}\right\rangle d \mathbb{P} & \leq \int \sum_{j=1}^{m}\left\|f_{n}^{j}\right\| \cdot\left\|g_{n}^{j}\right\| d \mathbb{P} \\
& \leq \int\left(\sum_{j=1}^{m}\left\|f_{n}^{j}\right\|^{q}\right)^{1 / q} \cdot\left(\sum_{j=1}^{m}\left\|g_{n}^{j}\right\|^{q^{\prime}}\right)^{1 / q^{\prime}} d \mathbb{P} \\
& \leq\left\|\left(\sum_{j=1}^{m}\left\|f_{n}^{j}\right\|^{q}\right)^{1 / q}\right\| \cdot\left\|\left(\sum_{r=1}^{m}\left\|g_{n}^{j}\right\|^{q^{\prime}}\right)^{1 / q^{\prime}}\right\|_{r^{\prime}} \\
& \leq 2 r^{\prime}\left(r^{\prime}-1\right)^{-1 / q}\left\|\left(\sum_{j=1}^{m}\left\|f_{n}^{j}\right\|^{q}\right)^{1 / q}\right\|_{r}\left\|\left(\sum_{j=1}^{m} \sum_{l=1}^{n}\left\|\varphi_{l, j}\right\|^{q^{\prime}}\right)^{1 / q^{\prime}}\right\|_{r^{\prime}},
\end{aligned}
$$

where the last inequality follows from [26, p. 418] and the triangle inequality.

Combining with (4.6), (4.7), (4.8) and note that $0<\varepsilon<1$ is arbitrary, then there exists a constant $K_{2}$ only depending on $X$ such that

$$
\left\|\left(\sum_{j=1}^{m} S_{q, n}^{q}\left(f^{j}\right)\right)^{1 / q}\right\|_{r} \leq K_{2} r^{\left(1+1 / q^{\prime}\right)}\left(r^{\prime}-1\right)^{-1 / q}\left\|\left(\sum_{j=1}^{m}\left\|f_{n}^{j}\right\|^{q}\right)^{1 / q}\right\|_{r} .
$$

Note here that $2 \leq r<\infty$, then it entails $r^{\prime\left(1+1 / q^{\prime}\right)}\left(r^{\prime}-1\right)^{-1 / q} \leq 4 r^{1 / q}$. Therefore, letting $K=4 K_{2}$ we have

for all $2 \leq r<\infty$. This completes our proof.

$$
\left\|\left(\sum_{j=1}^{m}\left\|f_{n}^{j}\right\|^{q}\right)^{1 / q}\right\|_{r} \leq K r^{1 / q}\left\|\left(\sum_{j=1}^{m} S_{q, n}^{q}\left(f^{j}\right)\right)^{1 / q}\right\|_{r},
$$

Corollary 4.3. A Banach space $X$ is linearly isomorphic to a Hilbert space if and only if the following two inequalities hold for $X$-valued dyadic martingales $f^{j}=\left(f_{n}^{j}\right)_{n=0}^{\infty}, j=1, \cdots, m$, there exists a constant $K$ only depending on $X$ such that

and

$$
\left\|\left(\sum_{j=1}^{m}\left\|f_{n}^{j}\right\|^{2}\right)^{1 / 2}\right\|_{r} \leq K r^{1 / 2}\left\|\left(\sum_{j=1}^{m} S_{2, n}^{2}\left(f^{j}\right)\right)^{1 / 2}\right\|_{r},
$$

for all $2 \leq r<\infty$.

$$
\left\|\left(\sum_{j=1}^{m} S_{2, n}^{2}\left(f^{j}\right)\right)^{1 / 2}\right\|_{r} \leq K r^{1 / 2}\left\|\left(\sum_{j=1}^{m}\left\|f_{n}^{j}\right\|^{2}\right)\right\|_{r}
$$


Proof. By the fact that Hilbert space is 2-uniformly smooth and 2-uniformly convex, then (4.9) and (4.10) follow from Theorem 4.1 directly. Hence, the "only if" part is proven.

For the "if" part, we assume that inequalities (4.9) and (4.10) hold true for $X$-valued dyadic martingales. Then, $X$ must have martingale type 2 and martingale cotype 2 . By a theorem of Kwapien, it follows that $X$ is linearly isomorphic to a Hilbert space.

\subsection{De la Peña-type inequalities for $p$-uniformly smooth space-valued martingales}

We conclude this paper by extending the De la Peña-type concentration inequalities for martingales with values in $p$-uniformly smooth space.

Recall that the following martingale inequality is known as the Freedman inequality, which was proved by Freedman [9] in 1975.

Theorem 4.4 (Freedman inequality). For every real-valued martingale $f=\left(f_{j}\right)_{j=0}^{\infty}$ with $\left|d_{j, f}\right| \leq 1$ a.s. for all $j \in \mathbb{N}$ and any positive numbers $a, b$. The following inequality holds

$$
\mathbb{P}\left\{f_{n}-f_{0} \geq r, s_{2, n}^{2}(f) \leq b \text { for some } n\right\} \leq\left(\frac{b}{r+b}\right)^{r+b} e^{r} \leq \exp \left\{-\frac{r^{2}}{2(r+b)}\right\}
$$

where $s_{2, n}^{2}(f)=\sum_{j=1}^{n} \mathbb{E}_{j-1}\left|d_{j, f}\right|^{2}$.

In order to avoid any boundness assumption on martingale differences, De la Peña [30] proved the following inequality for conditionally symmetric martingales.

Theorem 4.5 (De la Peña). Let $f=\left(f_{j}\right)_{j=0}^{\infty}$ a real-valued conditionally symmetric martingale. Then for $r, b>0$, we have

$$
\mathbb{P}\left\{f_{n}-f_{0} \geq r, S_{2, n}^{2}(f) \leq b \text { for some } n\right\} \leq \exp \left\{-\frac{r^{2}}{2 b}\right\} .
$$

Theorem 4.6 (De la Peña). Let $f=\left(f_{j}\right)_{j=0}^{\infty}$ be a real-valued conditionally symmetric martingale. Then, for $\beta, r, b>$ 0 and $\alpha \geq 0$, the following inequality holds

$$
\mathbb{P}\left\{\frac{f_{n}-f_{0}}{\alpha+\beta S_{2, n}^{2}(f)} \geq r, \frac{1}{S_{2, n}^{2}(f)} \leq b \text { for some } n\right\} \leq \exp \left\{-r^{2}\left(\frac{\beta^{2}}{2 b}+\alpha \beta\right)\right\} .
$$

By an argument from Pinelis [23] one can extend the Freedman inequality (Theorem4.4) and the De la Peña inequality (Theorem 4.5) to 2-uniformly smooth space valued martingales. Moreover, by applying the "martingale dimension reduction" argument (Lemma 3.2) one can further derive that Theorem 4.4 and Theorem 4.5 hold true for martingales with values in $p$-uniformly smooth space by replacing $S_{2, n}^{2}(f)$ to $S_{p, n}^{p}(f)$ of the left hand side and $r, b$ to $r^{p / 2}, K b$ of the right hand side respectively with $K=O\left(s_{p}(X)\right)$.

We now conclude this paper by extending the De la Peña inequality (Theorem 4.6) to $p$-uniformly smooth space-valued conditionally symmetric martingales.

Theorem 4.7. Let $f=\left(f_{j}\right)_{j=0}^{\infty}$ be an $X$-valued conditionally symmetric martingale (relatively to the filtration $\left.\left(\mathscr{F}_{j}\right)_{j=0}^{\infty}\right)$, where $X$ is a $p$-uniformly smooth space $(1<p \leq 2)$. Then, for each $\beta, r, b>0$ and $\alpha \geq 0$ the following inequality holds true,

$$
\mathbb{P}\left\{\frac{\left\|f_{n}-f_{0}\right\|}{\left(\alpha+\beta S_{p, n}^{p}(f)\right)^{2 / p}} \geq r, \frac{1}{S_{p, n}^{p}(f)} \leq b \text { for some } n\right\} \leq 4 \exp \left\{-r^{p}\left(\frac{\beta^{2}}{2 b K}+\frac{\alpha \beta}{K}\right)\right\},
$$

where $K=O\left(s_{p}(X)\right)$.

Proof. We prove the inequality for $\mathcal{H}$-valued conditionally symmetric martingales firstly and then apply the "martingale dimension reduction" argument (Lemma 3.2) to prove the case for $p$-uniformly smooth space-valued martingales. Without loss of generality, we assume that $f_{0}=0$ a.s. throughout the following proof.

Step I. Suppose that $f=\left(f_{j}\right)_{j=0}^{\infty}$ is a $\mathcal{H}$-valued conditionally symmetric martingale (relatively to the filtration $\left.\left(\mathscr{F}_{j}\right)_{j=0}^{\infty}\right)$, then applying Proposition 2.2 and $(3.4)$ of Theorem 3.1 , there exists another filtration $\left(\mathscr{G}_{j}\right)_{j=0}^{\infty}$ such that 
$f=\left(f_{j}\right)_{j=0}^{\infty}$ is a martingale and $g=\left(g_{j}\right)_{j=0}^{\infty}$ is a nonnegative super-martingale relatively to the filtration $\left(\mathscr{G}_{j}\right)_{j=0}^{\infty}$, where

$$
g_{n}=\frac{\cosh \lambda\left|f_{n}\right|}{\prod_{j=1}^{n} \exp \left\{\frac{\lambda^{2}\left|d_{j, f}\right|^{2}}{2}\right\}}, n \in \mathbb{N}_{0} \text { and } \lambda>0,
$$

Note that Hilbert space is 2-uniformly smooth, hence we now prove inequality 4.11) for $p=2$. Indeed, let $B=$ $\left\{\frac{\left|f_{n}\right|}{\alpha+\beta S_{2, n}^{2}(f)} \geq r, \frac{1}{S_{2, n}^{2}(f)} \leq b\right.$ for some $\left.n\right\}$ and define $\sigma=\inf \left\{n \in \mathbb{N}_{0}: \frac{\left|f_{n}\right|}{\alpha+\beta S_{2, n}^{2}(f)} \geq r, \frac{1}{S_{2, n}^{2}(f)} \leq b\right\}$. It is clear that $\sigma$ is a stopping time satisfying

$$
B=\left\{\frac{\left|f_{n}\right|}{\alpha+\beta S_{2, n}^{2}(f)} \geq r, \frac{1}{S_{2, n}^{2}(f)} \leq b \text { for some } n\right\}=\{\sigma<\infty\} .
$$

Then,

$$
\begin{aligned}
\mathbb{P}(B) & \leq 2 \int \frac{\cosh \frac{\lambda}{2}\left|f_{\sigma}\right|}{\exp \left\{\frac{\lambda r}{2}\left(\alpha+\beta S_{2, \sigma}^{2}(f)\right)\right\}} \cdot \mathbb{1}_{\{\sigma<\infty\}} d \mathbb{P} \\
& =2 \int \frac{\cosh \frac{\lambda}{2}\left|f_{\sigma}\right|}{\prod_{j=0}^{n} \exp \left\{\frac{\lambda^{2}\left|d_{\sigma, f}\right|^{2}}{4}\right\}} \cdot \exp \left\{\frac{\lambda^{2} S_{2, \sigma}^{2}(f)}{4}-\frac{\lambda r}{2}\left(\alpha+\beta S_{s, \sigma}^{2}(f)\right)\right\} \cdot \mathbb{1}_{\{\sigma<\infty\}} d \mathbb{P},
\end{aligned}
$$

for all $\lambda>0$.

By the Cauchy-Schwarz inequality and the fact that $\left(g_{j}\right)_{j=0}^{\infty}$ is a nonnegative super-martingale with $\mathbb{E}\left(g_{0}\right)=1$, we have

$$
\begin{aligned}
\mathbb{P}(B) & \leq 2 \inf _{\lambda>0}\left(\int \exp \left\{\frac{\lambda^{2} S_{2, \sigma}^{2}(f)}{2}-\left(\alpha+\beta S_{2, \sigma}^{2}(f)\right) \lambda r\right\} \cdot \mathbb{1}_{B} d \mathbb{P}\right)^{\frac{1}{2}} \\
& \leq 2\left(\int \exp \left\{-\frac{r^{2}\left(\alpha+\beta S_{2, \sigma}^{2}(f)\right)^{2}}{2 S_{2, n}^{2}(f)}\right\} \cdot \mathbb{1}_{B} d \mathbb{P}\right)^{\frac{1}{2}} \\
& \leq 2\left(\int \exp \left\{-\alpha \beta r^{2}-\frac{r^{2} \beta^{2} S_{2, \sigma}^{2}(f)}{2}\right\} \cdot \mathbb{1}_{B} d \mathbb{P}\right)^{\frac{1}{2}} \\
& \leq 2\left\{\exp -r^{2}\left(\alpha \beta+\frac{\beta^{2}}{2 b}\right)\right\}^{\frac{1}{2}} \cdot \mathbb{P}(B)^{\frac{1}{2}}
\end{aligned}
$$

Therefore,

$$
\begin{aligned}
\mathbb{P}(B) & =\mathbb{P}\left\{\frac{\left|f_{n}\right|}{\alpha+\beta S_{2, n}^{2}(f)} \geq r, \frac{1}{S_{2, n}^{2}(f)} \leq b \text { for some } n\right\} \\
& \leq 4 \exp \left\{-r^{2}\left(\frac{\beta^{2}}{2 b}+\alpha \beta\right)\right\} .
\end{aligned}
$$

Step II. We now turn to the case when $f=\left(f_{j}\right)_{j=0}^{\infty}$ is a conditionally symmetric martingale with values in $p$-uniformly smooth space. By Lemma3.2 there exists an $\mathbb{R}^{2}$-valued martingale $N=\left(N_{j}\right)_{j=0}^{\infty}$ (relatively to another filtration) such that
i) $\left\|f_{j}\right\|^{p} \leq\left|N_{j}\right|^{2}$
ii) $\left|N_{j}-N_{j-1}\right|^{2} \leq K_{1}\left\|d_{j, f}\right\|^{p}$,

for all $j \in \mathbb{N}$ with $K_{1}=O\left(s_{p}(X)\right)$. 
Furthermore, by the definition of $\left(N_{j}\right)_{j=0}^{\infty}$ (see (3.8)), we have for $j \in \mathbb{N}$

$$
\begin{aligned}
\left|N_{j}-N_{j-1}\right|^{2} & =\frac{p^{2}}{4} \cdot \frac{\left\langle J_{p}\left(f_{j-1}\right), f_{j}-f_{j-1}\right\rangle^{2} \cdot \mathbb{1}_{A_{j-1}}}{\left|N_{j-1}\right|^{2}}+(c+p)\left\|f_{j}-f_{j-1}\right\|^{p} \\
& \geq(c+p)\left\|f_{j}-f_{j-1}\right\|^{p}-\frac{p^{2}}{4} \cdot \frac{\left\|f_{j}-f_{j-1}\right\|^{2} \cdot \mathbb{1}_{A_{j-1}}}{\left\|N_{j-1}\right\|_{2}^{2\left(1-\frac{2}{p}\right)}} \\
& \geq\left(c+p-\frac{p^{2}}{4}\right)\left\|f_{j}-f_{j-1}\right\|^{p} \\
& \geq c\left\|f_{j}-f_{j-1}\right\|^{p},
\end{aligned}
$$

where $c=O\left(s_{p}(X)\right)$. The last inequality of (4.12) follows from the fact that $1<p \leq 2$.

Then, by using $i), i i)$ and (4.12), we have

$$
\begin{aligned}
& \mathbb{P}\left\{\frac{\left\|f_{n}\right\|}{\left(\alpha+\beta S_{p, n}^{p}(f)\right)^{2 / p}} \geq r, \frac{1}{S_{p, n}^{p}(f)} \leq b \text { for some } n\right\} \\
= & \mathbb{P}\left\{\frac{\left\|f_{n}\right\|^{\frac{p}{2}}}{\alpha+\frac{\beta}{K_{1}} K_{1} S_{p, n}^{p}(f)} \geq r^{\frac{p}{2}}, \frac{1}{c S_{p, n}^{p}(f)} \leq \frac{b}{c} \text { for some } n\right\} \\
\leq & \mathbb{P}\left\{\frac{\left|N_{n}\right|}{\alpha+\frac{\beta}{K_{1}} S_{2, n}^{2}(N)} \geq r^{\frac{p}{2}}, \frac{1}{S_{2, n}^{2}(N)} \leq \frac{b}{c} \text { for some } n\right\} \\
\leq & 4 \exp \left\{-r^{p}\left(\frac{\beta^{2}}{2 K b}+\frac{\alpha \beta}{K}\right)\right\},
\end{aligned}
$$

where $K=O\left(s_{p}(X)\right)$.

\section{Acknowledgements}

The author is partially supported by Natural Science Foundation of China (Grant No. 12071240). I would like to thank the Yau Mathematical Sciences Center of Tsinghua University for providing a great opportunity for me to complete this work. And I am grateful to the Associate Editor and the referees for their consideration.

\section{References}

[1] Ahlswede R, Winter A. Strong converse for identification via quantum channels[J]. IEEE Trans. Inf. Theory, 2002, 48(3): 569-579.

[2] Azuma K. Weighted sums of certain dependent random variables[J]. Tohoku Math. J., Second Series, 1967, 19(3): 357-367.

[3] Bañuelos R. A sharp good- $\lambda$ inequality with an application to Riesz transforms[J]. Mich. Math. J., 1988, 35(1): 117-125.

[4] Burkholder D L. Distribution function inequalities for martingales[J]. Ann. Probab., 1973: 19-42.

[5] Burkholder D L. Explorations in martingale theory and its applications[M]//École d'Été de Probabilités de SaintFlour XIX-1989. Springer, Berlin, Heidelberg, 1991: 1-66.

[6] Chidume C, Takens F. Geometric properties of Banach spaces and nonlinear iterations[M]. London, UK: Springer, 2009.

[7] Chung F, Lu L. Concentration inequalities and martingale inequalities: a survey[J]. Internet Math, 2006, 3(1): $79-127$.

[8] Ding J, Lee J R, Peres Y. Markov type and threshold embeddings[J]. Geom. Funct. Anal., 2013, 23(4): 1207-1229.

[9] Freedman D A. On tail probabilities for martingales[J]. Ann. Probab., 1975, 3(1): 100-118.

[10] Godbole, A.P., Hitczenko, P. Beyond the method of bounded differences, in: Microsurveys in Discrete Probability (Princeton, NJ, 1997), 41:43-58, 1998.

[11] Hayes T P. A large-deviation inequality for vector-valued martingales[J]. Comb. Probab. Comput., 2005. 
[12] Hoeffding W. Probability inequalities for sums of bounded random variables[J]. J. Am. Stat. Assoc., 1963, 58(301):13-30.

[13] Ishteva M. Properties and applications of the Caputo fractional operator. MSc thesis, Department of Mathematics, University of Karlsruhe, Karlsruhe, 2005.

[14] Kallenberg O, Sztencel R. Some dimension-free features of vector-valued martingales[J]. Probab. Theory Relat. Field, 1991, 88(2): 215-247.

[15] Klenke A. Probability theory: a comprehensive course[M]. Springer Science \& Business Media, 2013.

[16] Kwapień S, Woyczynski W A. Random series and stochastic integrals: single and multiple[M]. Birkhäuser, 1992.

[17] Ledoux M, Talagrand M. Probability in Banach Spaces: isoperimetry and processes[M]. Springer Science \& Business Media, 2013.

[18] Lieb E H. Convex trace functions and the Wigner-Yanase-Dyson conjecture[J]. Les rencontres physiciensmathématiciens de Strasbourg-RCP25, 1973, 19: 0-35.

[19] McDiarmid C. Centering sequences with bounded differences[J]. Comb. Probab. Comput., 1997, 6(1): 79-86.

[20] Naor A. On the Banach-space-valued Azuma inequality and small-set isoperimetry of Alon-Roichman graphs[J]. Comb. Probab. Comput., 2012, 21(4): 623-634.

[21] Oliveira R I. Concentration of the adjacency matrix and of the Laplacian in random graphs with independent edges[J]. arXiv preprint arXiv:0911.0600, 2009.

[22] Pinelis I. An approach to inequalities for the distributions of infinite-dimensional martingales[C]//Probability in Banach Spaces, 8: Proceedings of the Eighth International Conference. Birkhäuser, Boston, MA, 1992: $128-134$.

[23] Pinelis I. Optimum bounds for the distributions of martingales in Banach spaces[J]. Ann. Probab., 1994, 22(4): 1679-1706.

[24] Pipher J. Bounded double square functions[C]//Ann. Inst. Fourier. 1986, 36(2): 69-82.

[25] Pisier G. Martingales with values in uniformly convex spaces[J]. Isr. J. Math., 1975, 20(3-4): 326-350.

[26] Pisier G. Martingales in Banach spaces[M]. Cambridge University Press, 2016.

[27] Raginsky M , Sason I . Concentration of Measure Inequalities in Information Theory, Communications and Coding[C]// Concentration of Measure Inequalities in Information Theory, Communications, and Coding. Now Publishers Inc. 2012.

[28] Tropp J A. User-friendly tail bounds for sums of random matrices[J]. Found. Comput. Math., 2012, 12(4): 389434.

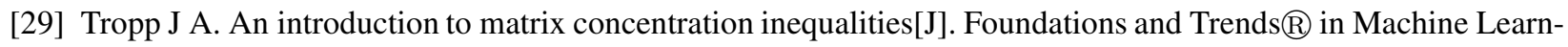
ing, 2015, 8(1-2): 1-230.

[30] Victor H. A general class of exponential inequalities for martingales and ratios[J]. Ann. Probab., 1999, 27(1): 537-564. 